\title{
REVIEWS
}

Check for updates

\section{Recovery from disorders of consciousness: mechanisms, prognosis and emerging therapies}

Brian L. Edlow $\mathbb{B}^{1,2}$, Jan Claassen ${ }^{3}$, Nicholas D. Schiff4 and David M. Greer $\mathbb{B}^{5 凶}$

Abstract | Substantial progress has been made over the past two decades in detecting, predicting and promoting recovery of consciousness in patients with disorders of consciousness (DoC) caused by severe brain injuries. Advanced neuroimaging and electrophysiological techniques have revealed new insights into the biological mechanisms underlying recovery of consciousness and have enabled the identification of preserved brain networks in patients who seem unresponsive, thus raising hope for more accurate diagnosis and prognosis. Emerging evidence suggests that covert consciousness, or cognitive motor dissociation (CMD), is present in up to $15-20 \%$ of patients with $\mathrm{DoC}$ and that detection of CMD in the intensive care unit can predict functional recovery at 1 year post injury. Although fundamental questions remain about which patients with DoC have the potential for recovery, novel pharmacological and electrophysiological therapies have shown the potential to reactivate injured neural networks and promote re-emergence of consciousness. In this Review, we focus on mechanisms of recovery from $\mathrm{DoC}$ in the acute and subacute-to-chronic stages, and we discuss recent progress in detecting and predicting recovery of consciousness. We also describe the developments in pharmacological and electrophysiological therapies that are creating new opportunities to improve the lives of patients with DoC.

Disorders of consciousness (DoC) are characterized by alterations in arousal and/or awareness, and common causes of DoC include cardiac arrest, traumatic brain injury (TBI), intracerebral haemorrhage and ischaemic stroke. The past several decades have witnessed major advances in our understanding of DoC, giving new hope for meaningful recovery in some patients. This new understanding has come via elucidation of the mechanisms underlying these disorders, increased accuracy of prognostication and the use of new therapeutic approaches. DoC exist on a temporal continuum ${ }^{1}$, and the principles and confounders of evaluation, prognostication and treatment change over time. The acute stage of DoC encompasses the time spent at the place where the injury occurred, in the emergency department and in the intensive care unit (ICU), whereas the subacute and chronic stages extend to time spent in inpatient rehabilitation hospitals, chronic nursing facilities and, for some patients, the home. The boundaries that delineate each stage of recovery are inherently arbitrary. Recent guidelines operationally define the acute period of $\mathrm{DoC}$ as the first 28 days after injury ${ }^{2}$, with the subacute-to-chronic period following thereafter.
In this Review, we discuss mechanisms of recovery from DoC and prognostication of outcome, as well as emerging treatments for patients along the entire temporal continuum of DoC. We consider these advances within the context of a clinical framework for classifying the behavioural features of DoC that has evolved over the past 50 years. In this framework, coma is defined as the complete absence of arousal and awareness ${ }^{3}$, the vegetative state (later renamed 'unresponsive wakefulness syndrome' (VS/UWS) $)^{4}$ is defined as arousal without awareness ${ }^{5,6}$ and the minimally conscious state (MCS) is defined as minimal, reproducible but inconsistent awareness 7 . Recently, MCS was sub-stratified into MCS without language (MCS-) and MCS with language $(\mathrm{MCS}+)^{8}$, a behavioural distinction that might have prognostic relevance ${ }^{9,10}$. The behavioural features of language expression and comprehension that distinguish MCS+ from MCS- include command-following, intelligible verbalization and intentional communication, the presence of any one of which is sufficient to indicate MCS $+{ }^{9}$. The 'upper bound' of DoC continues to be debated, but in this Review we consider patients who have emerged from MCS into a confusional state as still experiencing a disorder of consciousness, because a 


\section{Key points}

- A common pathophysiological mechanism underlying disorders of consciousness (DoC) is the withdrawal of excitatory synaptic activity across the cerebrum produced by deafferentation or disfacilitation of neocortical, thalamic and striatal neurons.

- Recovery from coma involves various mechanisms, culminating in the restoration of excitatory neurotransmission across long-range corticocortical, thalamocortical and thalamostriatal connections.

- The re-emergence of consciousness is associated with a shift in patterns of neuronal activity across the corticothalamic system that can be measured with EEG, PET or resting-state functional MRI.

- Task-based functional MRI and EEG can reveal cognitive motor dissociation in up to $15-20 \%$ of patients who seem unresponsive on behavioural examination, and emerging evidence suggests that early detection of cognitive motor dissociation in the intensive care unit predicts 1 -year functional outcomes.

- Amantadine is the only therapy that has been associated with the acceleration of recovery of consciousness in a randomized controlled trial of patients with subacute traumatic DoC, but multiple pharmacological and neuromodulatory therapies are now being tested.

- Emerging advances in diagnostic and prognostic techniques provide new opportunities to detect consciousness, monitor its recovery, elucidate its neuronal substrate and identify the therapeutic potential of promoting re-emergence of consciousness in a subset of patients with DoC.

\section{Disfacilitation}

The downregulation of

neuronal firing rates due

to deafferentation and/or

functional withdrawal of

excitatory neurotransmission confusional state is characterized by persistent dysfunction across multiple cognitive domains, behavioural dysregulation, symptom fluctuation, disorientation and, hence, altered consciousness.

A recent addition to the diagnostic classification scheme of patients with DoC is the concept of cognitive motor dissociation (CMD) ${ }^{11}$, also known as covert consciousness. CMD is characterized by volitional brain activity detected by task-based functional MRI (fMRI) or EEG in a patient whose bedside behavioural diagnosis suggests coma, VS/UWS or MCS-. Although many questions remain about the incidence, clinical characteristics, prognostic expectations and therapeutic responsiveness of patients with CMD, emerging evidence indicates that these patients represent a distinct subgroup of patients with DoC, whose brain networks and clinical features might fundamentally differ from those of other subgroups (FIG. 1). Therefore, we devote substantial space in this Review to the discussion of patients who are diagnosed with CMD in the acute and subacute-to-chronic stages of recovery.

We begin with a discussion of the state of the science regarding the common pathophysiological mechanisms that underlie DoC, as well as mechanisms of recovery that are shared between disease aetiologies. We consider how recent advances in behavioural examination, imaging and electrophysiology have improved the accuracy of prognostication. We focus not on prognostication of poor outcome but on the potential for recovery of consciousness. Although prognostication of poor outcome is important for decision-making, researchers have shifted their focus over the past decade to the study of patients who have unexpected recovery in the face of a previously forecast poor prognosis. These recoveries are alarming, as they reflect the risk of a self-fulfilling prophecy, wherein a poor prognosis leads to death via premature withdrawal of life-sustaining therapies ${ }^{12-14}$. However, unexpected recoveries also provide exciting opportunities, as we are increasingly able to detect consciousness, monitor its progress, identify its neuronal substrate and develop therapies to improve recovery. Hence, we focus on diagnostic tools that identify patients with a potential for recovery and personalized therapies that could promote this recovery. Finally, we highlight gaps in knowledge in the field of DoC and consider how rapid advances in diagnostic, prognostic and therapeutic modalities could fill these gaps to, ultimately, improve the lives of patients with DoC.

\section{Pathogenesis of DoC}

Cellular and circuit-based mechanisms. Coma is caused by several kinds of brain injury that can occur either alone or in combination. These insults are diffuse bihemispheric lesions ${ }^{15}$, bilateral lesions within the rostral paramedian brainstem ${ }^{16,17}$, bilateral diencephalon lesions with unilateral brainstem involvement ${ }^{18}$, or metabolic or toxic encephalopathies that produce widespread dysfunction of the corticothalamic system and its connections with the basal ganglia and limbic system ${ }^{15}$. Regardless of aetiology, the common pathophysiological mechanism underlying coma is broad withdrawal of excitatory synaptic activity across the cerebral cortex ${ }^{19,20}$. This downregulation of neuronal firing rates is produced by either direct structural loss of inputs or reduced input to neocortical and thalamic neurons, resulting in a process known as 'disfacilitation'19,21-23. Disfacilitation occurs as excitatory neurotransmission is withdrawn and the neuronal membrane potential passively hyperpolarizes owing to a dominance of potassium leakage currents. Under broad disfacilitation, as might occur in coma caused by diffuse injury or in the healthy brain under general anaesthesia, a very slow rhythm $(<1 \mathrm{~Hz})$ can arise across the corticothalamic system ${ }^{19,24}$.

Restoration of cerebral network activity. Both cellular and circuit mechanisms underlie recovery from coma, as excitatory neurotransmission is restored across corticocortical, thalamocortical and thalamostriatal connections $^{24-26}$. Thus, reversible coma can be the result of multiple mechanisms that globally alter neuronal function or disable specific circuits ${ }^{15}$. For structural brain injuries, one proposed mechanism of recovery the 'mesocircuit' model - focuses on the role of central thalamic neurons and their frontostriatal connections ${ }^{27}$ (BOX 1). In this model, restoration of function within the anterior forebrain mesocircuit is proposed to strongly covary with activation of the frontoparietal network, coalescing in a 'mesocircuit-frontoparietal' model for the graded return of behavioural responsiveness across different levels of $\mathrm{DoC}^{28}$.

The anterior forebrain mesocircuit ${ }^{25,27,29}$ and the frontoparietal network ${ }^{30,31}$ are consistently implicated in the restoration of cerebral activity during recovery from DoC. The anterior forebrain mesocircuit includes the frontal and prefrontal cortices and the striatopallidal negative feedback loop, which parallels the direct corticothalamic projections and influences thalamic outflow back to the cortex and striatum ${ }^{27}$ (BOX 1). This network of widespread anatomical connections made via the central thalamus makes the anterior forebrain 
Reafferentation

The re-establishment of afferent neuronal inputs, such as occurs during the process of neuronal plasticity that contributes to recovery of consciousness after a severe brain injury.

Deafferentation The disruption or disconnection of afferent neuronal inputs, such as when neuronal inputs in the setting of thalamic injury. cortical neurons lose their

mesocircuit vulnerable to multifocal brain injuries. The frontoparietal network comprises two subnetworks: the default mode network (DMN) and the executive control network. The DMN is anchored by midline nodes in the medial prefrontal cortex, posterior cingulate and precuneus, and mediates internal awareness or self-related processes $^{32,33}$. The executive control network is anchored by lateral nodes in the dorsolateral prefrontal and posterior parietal cortices, and mediates attention and environmental awareness ${ }^{34}$. The anterior forebrain mesocircuit and the frontoparietal network are interconnected, and the metabolic activity and functional connectivity within these networks increase as patients transition from coma to VS/UWS, MCS, confusional state and, ultimately, full cognitive recovery ${ }^{25,35-37}$. In studies in nonhuman primates, electrical stimulation of the central

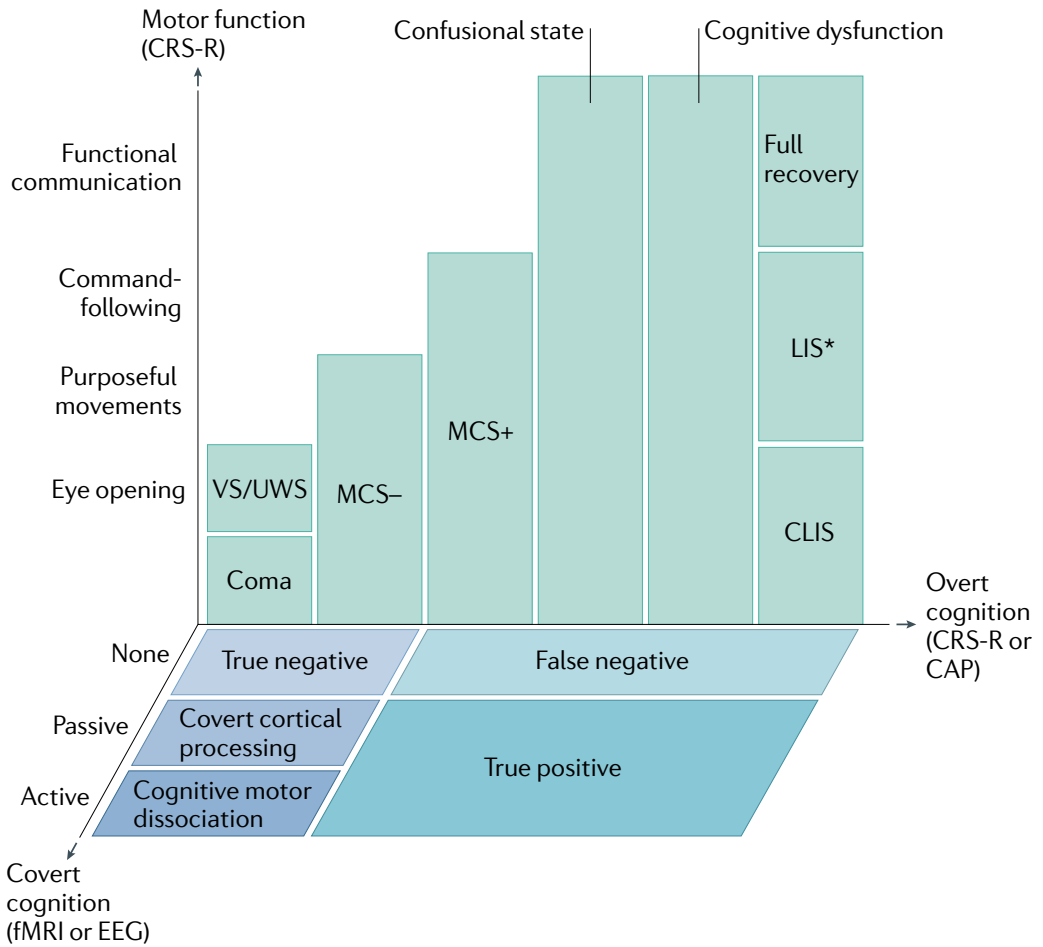

Fig. 1 | Multidimensional assessment of consciousness. During recovery from coma, patients are evaluated for overt cognition and motor function using the Coma Recovery Scale - Revised (CRS-R). This evaluation enables the classification of patients into the groups illustrated in green, with the exception of patients who emerge from a minimally conscious state with language (MCS+) to a confusional state, in whom the Confusion Assessment Protocol (CAP) is used to differentiate between a confusional state, cognitive dysfunction and full recovery. In patients with no behavioural evidence of language function, functional MRI (fMRI) or EEG evidence of command-following (active) indicates cognitive motor dissociation, $\mathrm{fMRI}$ or EEG responses within an association cortex during language or music stimuli (passive) indicate covert cortical processing, and an absence of $\mathrm{fMRI}$ or EEG responses indicates a true negative $\mathrm{fMRI/EEG} \mathrm{classification.} \mathrm{Patients} \mathrm{with}$ behavioural evidence of language are classified as false negatives if there are no fMRI or EEG responses, and as true positives if there are $\mathrm{FMRI}$ and EEG responses. CLIS, complete locked-in syndrome; LIS, locked-in syndrome; MCS-, minimally conscious state without language; VS/UWS, vegetative state/unresponsive wakefulness syndrome. *Patients with LIS are identified by the presence of consistent purposeful movements, typically vertical eye movements, and a reliable movement-based communication system. Patients with LIS who demonstrate inconsistent movements would not be distinguishable from patients with CLIS, cognitive dysfunction, confusional state or MCS. Some patients with LIS are able to communicate via assistive communication devices. Adapted with permission from REF. ${ }^{169}$, OUP. lateral thalamic nucleus selectively produced arousal from anaesthesia-induced coma $^{38,39}$, providing strong evidence that the anterior forebrain mesocircuit and the frontoparietal network are linked via this brain region.

The central thalamus is also the primary thalamic target of projections from the brainstem arousal nuclei ${ }^{40,41}$. Recovery of consciousness depends upon the functional re-emergence of the brainstem's ascending arousal network ${ }^{42}$, also known as the ascending reticular activating system ${ }^{43,44}$, which must provide sufficient input to the anterior forebrain mesocircuit and frontoparietal network to depolarize neocortical neurons and facilitate firing. Identification of the specific nodes and connections of the ascending arousal network, mesocircuit and frontoparietal network that are essential for recovery of consciousness will require further experimental and clinical investigation. Furthermore, it is possible that the co-activation of the mesocircuit and frontoparietal network by the ascending arousal network is not sufficient to generate conscious awareness without the re-emergence of additional cortical networks ${ }^{45}$.

When functional reafferentation occurs during recovery, the resting membrane potentials of neocortical neurons are gradually restored, that is, they become more depolarized, resulting in changes to neuronal firing patterns that are reflected in the $\mathrm{EEG}^{46}$. Similar EEG patterns are observed during emergence from general anaesthesia ${ }^{24}$. The overall frequency content of the EEG is indicated by the power spectrum, which indexes the contribution of oscillations at different frequencies within the EEG signal ${ }^{47}$. One model of neuronal recovery, known as the 'ABCD' model, organizes these sequential changes in EEG power spectra into four coarse-grained (or 'widely separated') categories, which are hypothesized to reflect the severity of thalamocortical deafferentation (TABLE 1). This model can be used to understand the varying levels of structural or functional deafferentation that occur in patients with DoC. Notably, a behavioural diagnosis can be associated with more than one spectral category.

The first category in the ABCD model, 'A', resembles the experimental 'cortical slab' preparation ${ }^{20}$, in which neocortical neurons have marked membrane hyperpolarization and the EEG power spectrum is restricted to $<1 \mathrm{~Hz}$. A-type dynamics can arise when the neocortex is completely or almost completely deafferented owing to structural injuries ${ }^{22}$. For example, some patients with chronic VS/UWS resulting from severe hypoxic-ischaemic encephalopathy (HIE) display an A-type power spectrum ${ }^{48}$. Some evidence suggests that A-type dynamics, which arise in healthy human and animal brains under anaesthesia ${ }^{19,24}$, are neuroprotective and driven by mechanisms that evolved to preserve brain volume and promote recovery following injury ${ }^{49,50}$.

When membrane potentials are depressed, depolarization of neocortical neurons can result in spontaneously generated bursting for seconds at a rate of $\sim 5-9 \mathrm{~Hz}$, as a result of intrinsic membrane properties ${ }^{51}$, producing a narrow oscillation in the same frequency range in the $\mathrm{EEG}^{29}$, categorized as 'B-type' dynamics. Partial restoration of neocortical membrane potentials and coincident bursting of deafferented thalamic neurons 
Dynamic range

In neocortical neurons, the nominal span of the absolute firing rate, specific patterns of firing and the differential ability of individual neurons to integrate synaptic information, all of which vary as a function of membrane potential that is, in turn, controlled by background synaptic input.

during wakefulness generate oscillations of $\sim 3-7 \mathrm{~Hz}$ with coupled higher frequency rhythms in the EEG ${ }^{52,53}$. This 'C-type' pattern is thus predicted to appear in the setting of more preserved cerebral metabolism ${ }^{54}$. In the healthy intact cerebral cortex, restoration of the normal EEG power spectrum with a peak in the alpha frequency range $(8-13 \mathrm{~Hz})$ and peaks in higher frequency ranges are associated with normal neocortical neuronal firing patterns, or 'D-type' dynamics ${ }^{55}$. Physiological correlates, in the form of shifts from B-type to C-type or D-type dynamics, associated with the transition from VS/UWS to MCS and higher levels of recovery, have been seen in some medication-responsive patients ${ }^{29}$ and in patients who show spontaneous recovery during the acute ${ }^{56,57}$

\section{Box 1 | A mesocircuit model of recovery of consciousness}

All severe brain injuries that cause coma share a common pathological substrate, which is a marked loss of synaptic background activity owing to either widespread neocortical, striatal and thalamic neuronal death, dysfunction or disconnection, or focal injuries to the paramedian mesodiencephalon (that is, the central thalamus and rostral brainstem tegmentum). Widespread disfacilitation occurs, involving neocortical, striatal and thalamic neurons, with a specific contribution from central thalamic neurons that integrate loss of input across multiple cerebral targets. Loss of medium spiny neuron inhibition of the globus pallidus interna (GPi) produces active inhibition of components of the central thalamus, including the central lateral nucleus (CL; see the figure). Collectively, these mechanisms are proposed to produce a downregulation of activity across the anterior forebrain, resulting in limited or fluctuating behavioural responsiveness ${ }^{27}$. This anatomical foundation supports the functional activation of the frontal cortex and striatum (Str) with direct stimulation of the CL using electrical ${ }^{290}$ or optogenetic ${ }^{291}$ techniques. Restoration of function across this network is associated with a shift in neuronal firing patterns across the corticothalamic system that can be measured in the spatiotemporal dynamics of the electroencephalogram or changes in the differential ability of individual neurons to integrate synaptic information controlled by background synaptic input ${ }^{200,292}$. However, normalization of resting EEG background activity might not correlate with full restoration of cognitive function following coma, as deficits in resource allocation and a failure to recruit the full dynamic range of neocortical neurons have a key role in functional outcome.

The figure shows the mesocircuit model superimposed upon a sagittal image of an ex vivo human brain specimen scanned at a resolution of $100 \mu \mathrm{m}$. Putative sites of action within the mesocircuit for pharmacological and non-pharmacologic therapies ${ }^{293}$ are indicated by blue arrows. PTg, pedunculotegmental nucleus; Ret, reticular nucleus of the thalamus. Figure adapted from REF. ${ }^{294}$, CC BY 4.0 (https://creativecommons.org/ licenses/by/4.0/).

Transcranial direct current stimulation Transcranial magnetic stimulation

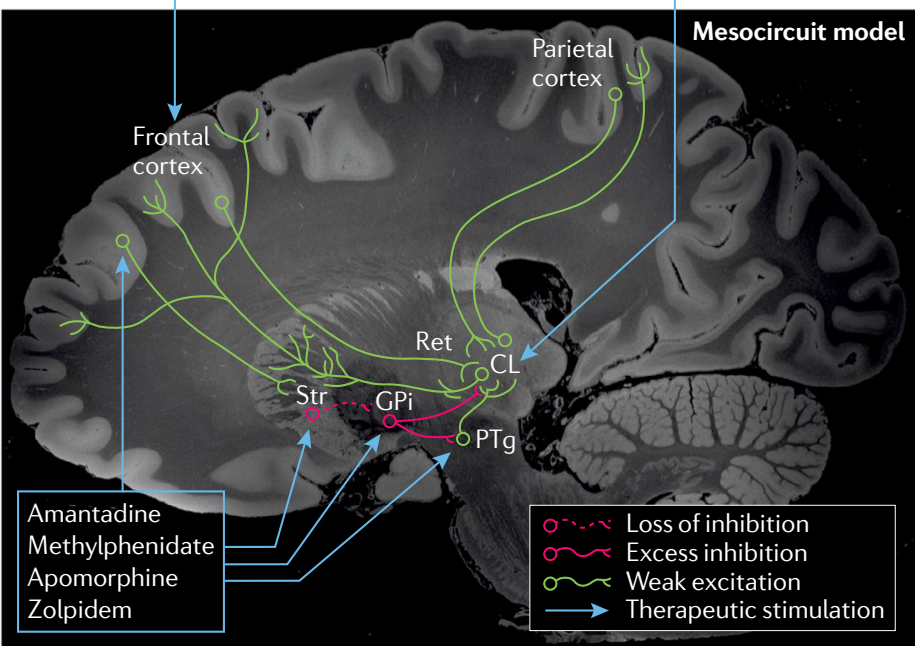

or subacute-to-chronic ${ }^{54}$ stage of DoC. Recovery from VS/UWS or MCS to a confusional state or higher levels of cognitive function is typically associated with restoration of D-type dynamics ${ }^{58,59}$. Importantly, several specific predictions of the mesocircuit model and the ABCD model have been verified in groups of patients with acute and subacute-to-chronic DoC resulting from traumatic and non-traumatic causes ${ }^{25,29,54,56,57,60}$.

In patients who recover to the confusional state, restoration of tonic firing in the thalamus and D-type dynamics can coexist with local electrophysiologic abnormalities, for example, increased delta to alpha power ratios ${ }^{59}$. Patients who recover full consciousness can experience persistent cognitive dysfunction, owing to impaired arousal regulation from the anterior forebrain mesocircuit ${ }^{58}$. Ongoing neuronal dysfunction in these patients is proposed to result from restriction of the dynamic range of neocortical pyramidal neurons in the frontoparietal network and their loop connections with the basal ganglia and thalamus; evidence for a key role of the central thalamus in this restriction is accumulating ${ }^{38,39}$. In the healthy awake brain, neocortical neurons are in a 'high conductance' state ${ }^{61}$ and exhibit a flexible, dynamic repertoire of firing motifs ${ }^{55}$. Although direct measurements are as yet unavailable, we anticipate a failure to restore the full dynamic range of neocortical and striatal neurons in patients with confusion and cognitive impairments ${ }^{62}$.

\section{Acute disorders of consciousness}

As mentioned above, acute DoC have various aetiologies, including toxic-metabolic insults and structural lesions, which can result from TBI, global HIE from cardiac arrest, ischaemic stroke, intracerebral haemorrhage, subdural haemorrhage, epidural haemorrhage and subarachnoid haemorrhage (SAH). DoC in cardiac arrest result primarily from bihemispheric dysfunction because the brainstem is typically resistant to anoxic injury in all but the most severe cases ${ }^{63}$. Acute DoC after cardiac arrest can also be caused by various factors, including seizures, cerebral oedema, metabolic abnormalities and sedating medications ${ }^{15}$. Thus, in these patients, eliminating diagnostic confounders, using electrophysiological markers to evaluate electrical dysfunction and using imaging markers to assess structural injury are of paramount importance.

In contrast to HIE, acute DoC after TBI can result from heterogeneous, multifocal injuries to the cerebral hemispheres and brainstem, making acute prognostication far more challenging. Furthermore, delayed recovery is more common after TBI than after $\mathrm{HIE}^{64}$, and prolonged observation and therapy can reveal remarkable recovery in some patients with DoC after TBI for whom recovery seemed unlikely early on in their illness ${ }^{65-68}$. Ischaemic stroke follows a more predictable pattern than TBI, with swelling typically maximal around 3-5 days after infarction ${ }^{69}$, which enables more reliable prognostication. In slight contrast, oedema resulting from intracerebral haemorrhage can occur very early or after a delay, and can sometimes be unexpectedly prolonged $^{70}$. Finally, SAH can be dynamic, with different insults occurring within the first few hours (for example, hydrocephalus and increased intracranial pressure), 
Table 1 | The 'ABCD' model of corticothalamic dynamics

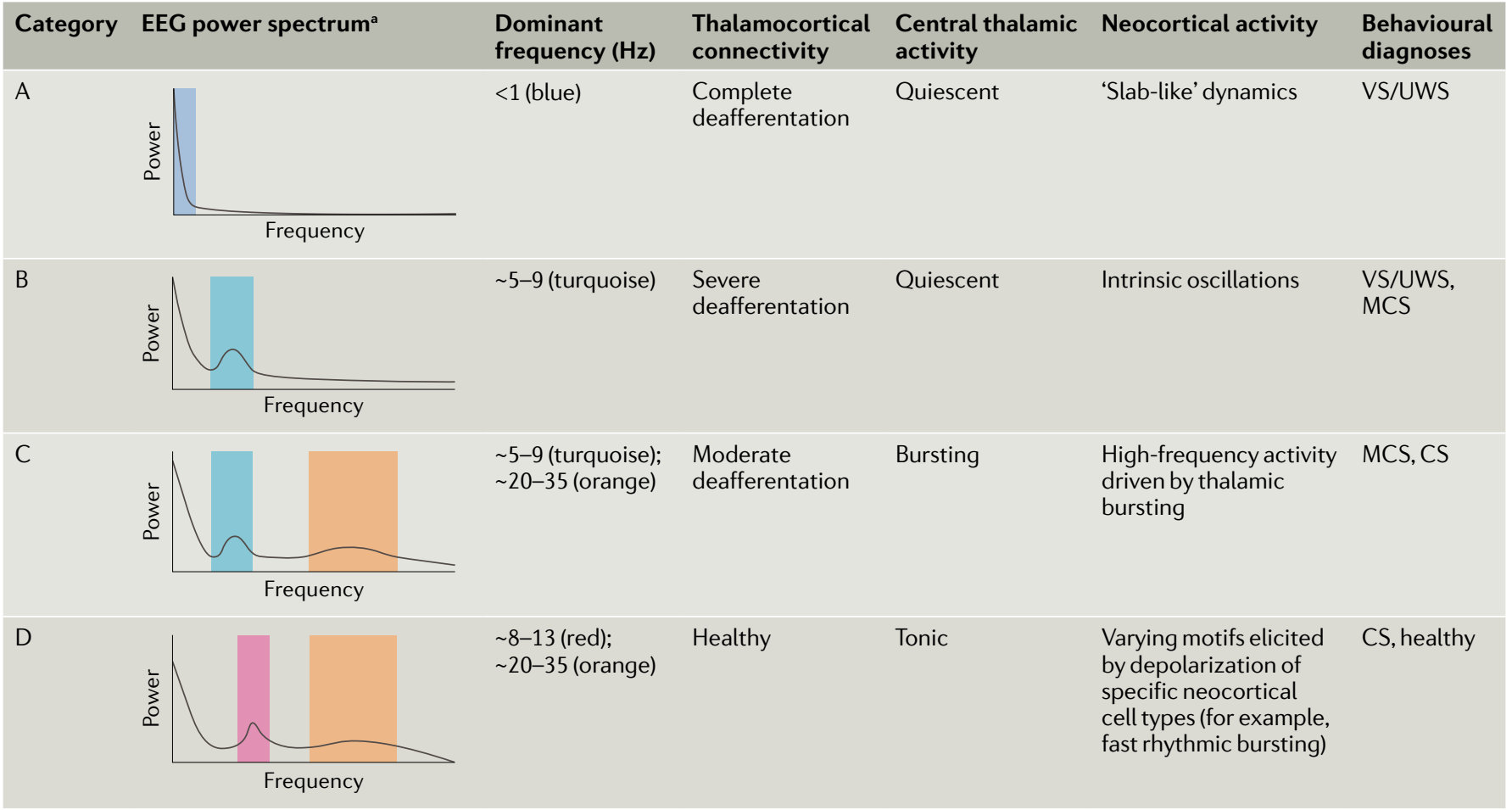

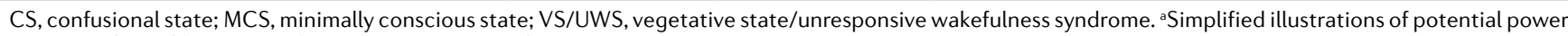
spectra. Adapted from REF."7 , Springer Nature Limited.

days (for example, cerebral vasospasm) and weeks (for example, seizures), making prognostication particularly challenging ${ }^{71}$. These different aetiologies all result in structural injury, cerebral oedema, electrical dysfunction and increased susceptibility to toxic-metabolic effects.

Accurate prognostication of $\mathrm{DoC}$ requires assessment of the primary brain injury and concomitant injuries or organ dysfunction, as well as pre-existing comorbidities, such as dementia or cardiac, pulmonary or hepatic dysfunction. Prognostication of poor outcome has dominated the field to date, leading to multiple prognostic guidelines for the various disease states ${ }^{72,73}$. Accurate prediction of poor outcome is important, as withdrawal of life-sustaining therapies is a leading cause of death for unconscious patients with acute brain injury ${ }^{14,74-76}$. Therefore, an inaccurate, overly pessimistic prediction could result in premature withdrawal of life-sustaining therapies, leading to death in patients who might have reached acceptable outcomes if given sufficient time to recover ${ }^{12,13}$. However, the process of determining poor outcome does not, by its converse, leave as its remainder those patients who will necessarily have a good outcome, or even recover consciousness. Accurate prognostication of poor outcome necessarily emphasizes high specificity, that is, a low rate of false positive predictions, over sensitivity. This is likely to leave a group of patients without a specific prediction of poor outcome but who, ultimately, show a wide range of outcomes, from chronic VS/UWS to full recovery.

\section{Detection and prediction of recovery}

Clinical examination. The neurological examination remains at the core of acute evaluation and prognostication of patients with DoC in the ICU. This examination is non-invasive, can be performed serially by numerous types of examiners and has been the subject of recent advances in technology, such as automated pupillometry. However, neurological examination is also susceptible to confounding by factors such as psychoactive medications, toxic-metabolic disturbances and variations in temperature - both hyperthermia and hypothermia. Hypothermia, in particular, affects drug metabolism, thus further confounding the examination. Clinical scoring systems used in these examinations include the Glasgow Coma Scale ${ }^{3}$ and the Full Outline of UnResponsiveness $(\text { FOUR })^{77}$, with the latter showing potential advantages over the former for the prediction of in-hospital mortality and functional outcomes ${ }^{78}$. For patients with $\mathrm{SAH}$, the World Federation of Neurosurgical Societies (WFNS) grading scheme is used ${ }^{79}$. This scale is most commonly assessed on admission, when the examination might be affected by sedation or untreated hydrocephalus, potentially leading to an inaccurate, overly pessimistic prognostication. Serial assessment with the WFNS score after elimination of confounders can reveal improvement, indicating a better chance of recovery than that predicted by the initial assessment ${ }^{80}$. The Pittsburgh Cardiac Arrest Category score is used for prognostication in patients with post-cardiac arrest DoC and incorporates cardiovascular and respiratory organ function data into the prognostic assessement ${ }^{81}$. Use of scoring systems in the acute ICU setting provides an objective, cross-sectional measure of the overall state of a patient, taking into account not just neurological but also general critical care factors ${ }^{82}$. This scoring can also be performed serially to detect improvement or deterioration. 
The neurological examination begins with an assessment of consciousness, in which the response of the patient to maximal noxious stimulation (auditory, visual and tactile) is assessed. Then, brainstem functions are tested. The function of the upper brainstem, specifically the pupillary and corneal reflexes, has prognostic relevance in patients with cardiac arrest ${ }^{72}$ and $\mathrm{TBI}^{83}$. The pupillary light reflex assesses the function of afferents and efferents of the midbrain, and absence of this reflex $>72 \mathrm{~h}$ post arrest (or longer if confounding from hypothermia and/or drug metabolism is possible) has been almost uniformly associated with poor outcome, defined as severe disability, VS/UWS or death ${ }^{84}$. However, the presence of the pupillary light reflex does not guarantee a good outcome. Clinician technique is important, as subtle changes in pupil size could be missed with the naked eye, and we recommend the use of a bright LED light and a magnifying glass. Automated pupillometry can complement the manual approach and can measure not only the presence or absence of reactivity but additional metrics including the speed of constriction ${ }^{85,86}$.

The corneal reflex tests pontine function through cranial nerves V and VII, and is of particular prognostic importance owing to the anatomical proximity of its pathway to pontine arousal nuclei ${ }^{41}$; however, prognostication using this reflex is also subject to vagaries in testing. A recent worldwide survey of intensivists and neurologists found that clinicians commonly test for a response too far laterally on the sclera and use submaximal stimulation, both of which could lead to a falsely negative (absent) reflex response ${ }^{87}$. Corneal sensitivity is highest at the edge of the iris and decreases further out on the conjunctiva ${ }^{88}$. Furthermore, although use of a liquid stimulus, for example, a squirt of sterile saline onto the cornea, might be a useful screening test, more potent stimulation is required if the reflex is found to be absent. We suggest the use of light pressure adjacent to the iris with a cotton-tipped applicator. These examination techniques and limitations should be kept in mind when considering any false positive results from corneal or pupillary testing that are reported in the literature, as details of the technique used are commonly absent from these publications.

Additional reflexes that can be used to test brainstem function include the oculocephalic reflex (doll's eyes reflex), which should only be tested in patients with known cervical spine stability, and the oculovestibular reflex (tested using cold calorics), which should only be tested in patients with integrity of the auditory canal and tympanic membrane ${ }^{15}$. Both reflexes test pontine and midbrain function. Lower brainstem dysfunction, tested through the cough and gag reflexes, is observed in the most severe brain injuries, which often involve herniation ${ }^{15}$.

After cardiac arrest, a motor score of 1 (no movement) or 2 (extensor posturing) previously connoted a uniformly poor outcome, but recent studies report that good outcomes can occur in a small, but not negligible, proportion of patients with these scores ${ }^{89}$. The most recent European guideline ${ }^{90}$ suggest using the motor score only for screening patients after cardiac arrest to identify those at risk of poor outcome, but even this seems superfluous. The presence of a motor score of 4 (withdrawal from pain) or higher usually indicates a patient destined for a good outcome ${ }^{91}$.

The most comprehensive, evidence-based behavioural assessment for detecting signs of consciousness in patients recovering from coma is the Coma Recovery Scale - Revised (CRS-R ${ }^{92}$. This scale has been found to detect evidence of volitional responses, such as gaze tracking, in $\sim 40 \%$ of patients previously diagnosed with VS/UWS on the basis of the consensus opinion of treating clinicians ${ }^{93}$. Early detection of consciousness has prognostic relevance ${ }^{94-96}$ and is a primary determinant in goals of care decisions ${ }^{97}$. The drawback to the CRS-R is that it is time-intensive, as the assessment can take up to $30-40 \mathrm{~min}$. Therefore, the CRS-R is often not practical as a daily assessment tool for patients in the ICU who are unable to tolerate being off sedation for prolonged periods of time. By contrast, the FOUR score has been validated for use across a spectrum of diseases in the ICU setting, is reproducible and can be performed quickly, making it an optimal tool for use in critically ill patients with brain injuries ${ }^{77,78}$.

Imaging. Brain imaging is an essential component of the acute assessment of DoC. Head CT is the imaging modality that is most commonly used in this setting, as it is widely accessible and enables rapid data acquisition. CT might be useful for predicting early mortality in some patients with acute DoC, such as when global oedema is detected in patients with cardiac arrest ${ }^{98}$ or $\mathrm{SAH}^{99}$. However, CT has a limited sensitivity for detecting many coma-causing entities, including traumatic axonal injury and HIE, and thus is rarely used in isolation to predict outcomes in patients with acute DoC.

Compared with CT, MRI substantially enhances the detection of coma-causing lesions ${ }^{100}$, particularly within the brainstem ${ }^{101}$, providing a clearer picture of an individual patient's potential for recovery. For families and clinicians facing time-sensitive decisions about continuing life-sustaining therapy for patients in the ICU, MRI data often factor heavily in discussions about the potential for recovery ${ }^{97}$. For example, evidence indicates that diffusion restriction on diffusion-weighted imaging can predict functional outcome in patients with cardiac arrest ${ }^{102-104}$, albeit with lower specificity after hypothermia ${ }^{91}$. Other evidence suggests that microbleeds detected with susceptibility-weighted or $\mathrm{T} 2{ }^{*}$-weighted imaging can predict coma duration ${ }^{105,106}$ and functional outcomes ${ }^{105,107,108}$ in patients with severe TBI.

Despite these promising findings, clinicians must consider the potential limitations and confounders of MRI data when incorporating them into prognosis discussions. For example, in patients with severe TBI, diffusion restriction on diffusion-weighted imaging does not invariably represent irreversible axonal injury, as multiple studies have reported recovery of white matter integrity, and corresponding behavioural recovery, in patients with this MRI finding ${ }^{65,67}$. Similarly, the results of radiological-pathological correlation studies indicate that axons can be preserved at sites of microhaemorrhage in patients with severe $\mathrm{TBI}^{107,109}$, suggesting that 
microbleeds do not indicate irreversible haemorrhagic axonal injury in these individuals. These observations provide a potential pathophysiological basis for the reports of unexpected recovery in patients with extensive microhaemorrhages, including in the brainstem ${ }^{65,66,108,110}$. Avoiding misinterpretation of neuroimaging data and recognizing reversible changes are essential for accurate prognostication of patients with DoC. Importantly, the identification of these reversible changes suggests that the time window for therapeutic intervention is longer than was previously assumed, which has implications for the planning of future trials of neuroprotective therapies $^{111,112}$.

The limitations of MRI are a primary motivator for the deployment of advanced imaging techniques, which have begun to show promise for improving the accuracy of outcome prediction in patients with $\mathrm{DoC}^{113-117}$. To date, the most compelling data come from two studies that have shown a high sensitivity and specificity of diffusion tensor imaging (DTI) measurements for predicting functional recovery in patients with HIE ${ }^{118}$ and $\mathrm{TBI}^{119}$. DTI measures the directional diffusion, or fractional anisotropy, of water molecules along axonal bundles, providing an inferential measure of axonal integrity ${ }^{120}$. DTI measures of fractional anisotropy within whole-brain white matter predicted 6-month outcomes in patients with acute $\mathrm{HIE}^{118}$, and measures of fractional anisotropy within cortical and subcortical white matter bundles predicted 1-year outcomes in patients with acute $\mathrm{TBI}^{119}$, suggesting that DTI could contribute to early prognostication of DoC.

High-resolution diffusion MRI techniques can also map the structural connectivity of the brain networks that support re-emergence of consciousness by reconstructing axonal fibre tracts. Diffusion MRI tractography detects acute white matter injury ${ }^{121-123}$, and evidence indicates that this technique can be used in the ICU to predict long-term cognitive outcomes in patients with acute $\mathrm{TBI}^{121}$. In addition, tractography can be used to map the ascending arousal network in patients with acute $\mathrm{DoC}^{42,109,124}$ (FIG. 2). This approach could be used to identify preserved subcortical connections that might support recovery or provide a target for therapeutic stimulation ${ }^{124}$. Given the emerging evidence that quantitative diffusion MRI techniques could enhance the accuracy of outcome prediction, efforts are underway to standardize data acquisition methods ${ }^{125-127}$, disseminate open-source analytic code ${ }^{128}$ and promote widespread access to these prognostic tools.

A second motivation for the use of advanced imaging techniques in patients with acute $\mathrm{DoC}$ is the emergence of a new network-based conceptual framework ${ }^{129,130}$ for studying coma pathogenesis and recovery, which is replacing the classic lesion-based framework ${ }^{131-133}$ (FIG. 3). Recovery of consciousness is now conceptualized as being contingent upon the re-emergence of dynamic interactions between multiple subcortical and cortical networks, including the ascending arousal network, mesocircuit and frontoparietal network discussed above. By providing a personalized brain connectivity map, or connectome, for each patient, it is possible that advanced MRI techniques will reveal the network architecture that underlies synaptic plasticity and circuit reorganization as patients recover consciousness ${ }^{134,135}$.

The DMN is the most frequently studied network in acute DoC because of its well-established contributions to conscious awareness ${ }^{136}$. Initial evidence from resting-state fMRI studies of patients with cardiac arrest, TBI and coronavirus disease 2019 (COVID-19) suggests that DMN functional connectivity is necessary, but not sufficient, for recovery of consciousness ${ }^{36,114,115,117,137,138}$. Additional contributions to recovery are believed to emerge from the salience network, which comprises anterior insular, dorsal anterior cingulate and frontoinsular nodes, and has robust connectivity to subcortical and limbic structures, and from the executive control network, which comprises dorsolateral frontal and parietal nodes, as described above ${ }^{34}$. Identifying the combinations of additional networks beyond the DMN, salience network and executive control network that are required for recovery of consciousness is an area of active study.

Electrophysiology. Complementary to clinical examination and neuroimaging, numerous electrophysiological techniques are used in the ICU to detect preserved brain networks, support prognostication and guide therapy ${ }^{139}$. These techniques either record the electrical activity of the resting brain, for example, classic EEG, or record an electrical signal from the brain in response to perturbation of the brain, such as that caused by auditory stimulation (for example, event-related potentials (ERPs)), peripheral electrical or tactile stimulation (for example, somatosensory evoked potentials), or direct electrical or magnetic stimulation of the brain (for example, transcranial magnetic stimulation (TMS)).

Electrographic seizures and status epilepticus are common causes of altered consciousness in patients in the ICU ${ }^{140,141}$. The vast majority of seizures in patients with acute DoC are 'non-convulsive', that is, not associated with clinically detectable motor manifestations $\mathbf{s}^{141,142}$. Determining the extent to which consciousness is altered by non-convulsive seizures versus the underlying brain injury can be challenging. Multiple EEG patterns, including seizures and periodic discharges, are associated with poor outcome $e^{143-148}$, but whether these patterns are surrogate markers of brain injury or are causing secondary brain injury, or both, is unclear. Some EEG features, such as the presence of sleep architecture and reactivity to external stimuli, including painful tactile stimulation, are associated with good outcomes, particularly after cardiac arrest ${ }^{149,150}$. The temporal evolution of EEG features has not been not well-studied, but could parallel clinical recovery.

Particular note should be made of the prognostic relevance of myoclonic status epilepticus (MSE) in patients with post-cardiac arrest DoC. Despite its name, the diagnosis of MSE is not based on EEG but, rather, is a clinical manifestation of myoclonic movements involving the face, trunk and limbs ${ }^{72}$. MSE was traditionally considered to be associated with poor outcome ${ }^{72}$, but more recent data show that some patients with MSE who are treated aggressively, such as with therapeutic temperature modulation and anti-seizure medications, 

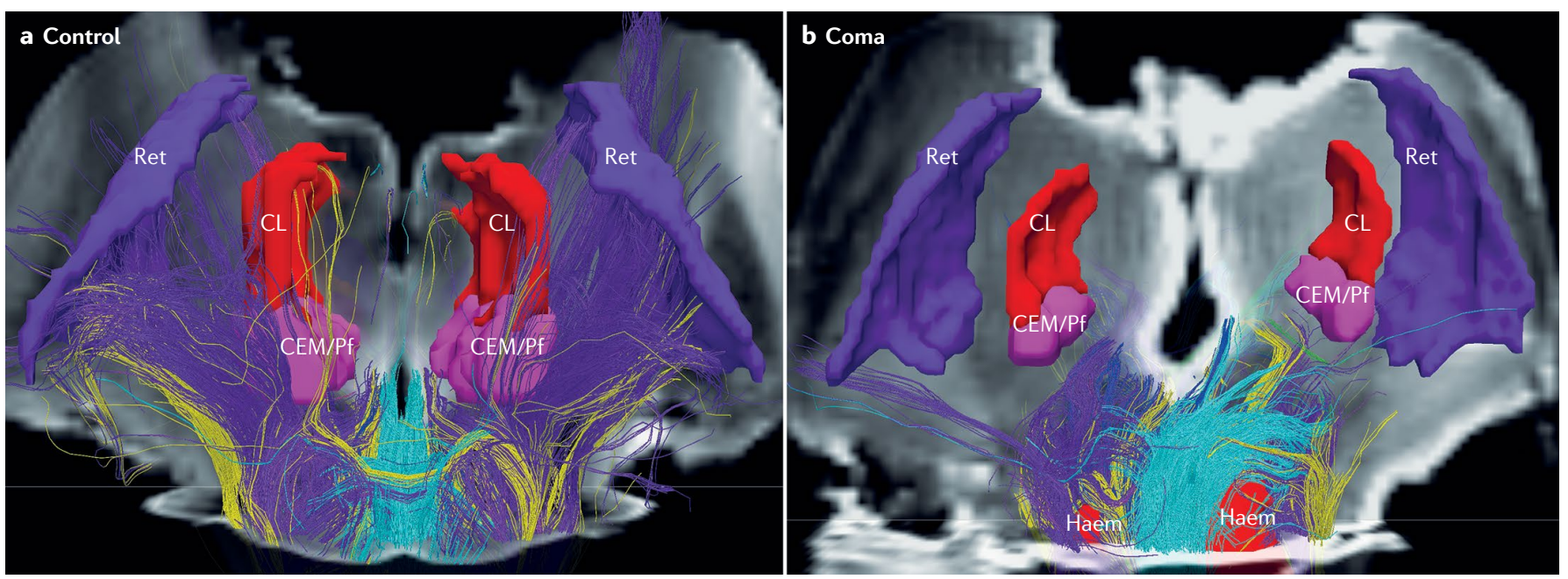

c
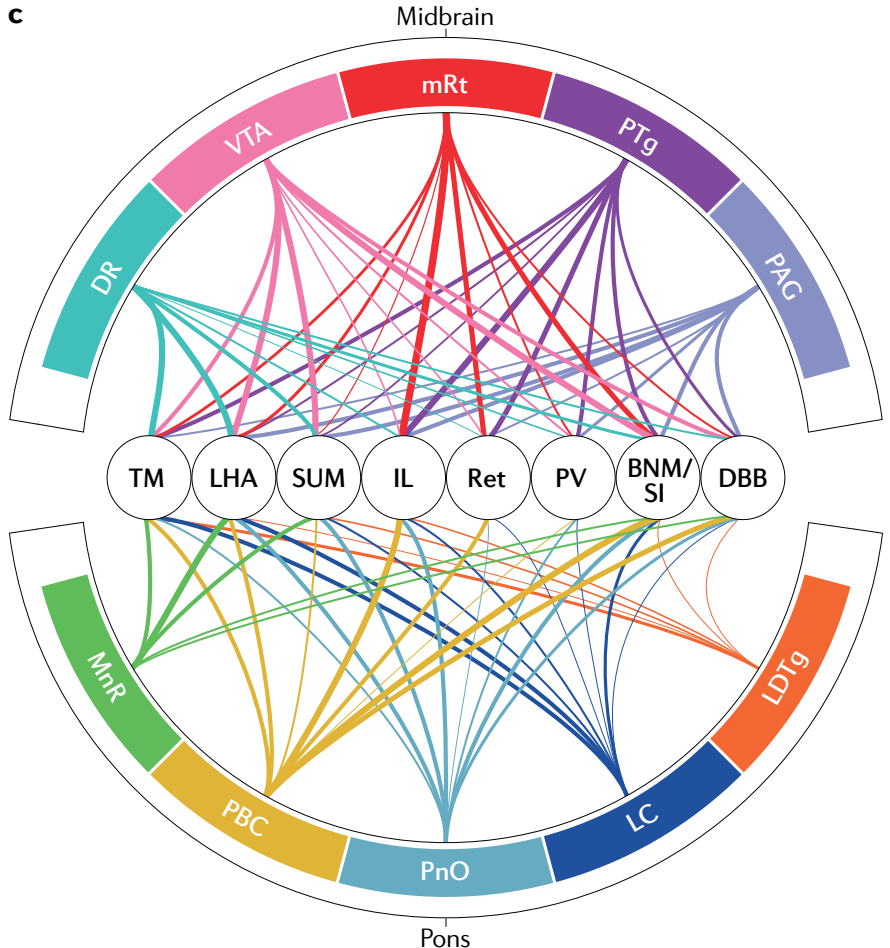

Fig. 2 | Diffusion tractography detects acute disconnection of the ascending arousal network. a,b | Post-mortem diffusion tractography scans showing that the brainstem and thalamic nuclei of the ascending arousal network are extensively interconnected in a control individual (part a), but completely disconnected in a patient who died in the intensive care unit 3 days after a coma-inducing severe traumatic brain injury (part b). Scans shown from a posterior perspective, with fibre tracts colour-coded according to the brainstem nucleus from which they originate: purple, pedunculotegmental nucleus (PTg); yellow, parabrachial complex (PBC); turquoise, dorsal raphe (DR); dark blue, locus coeruleus (LC); green, median raphe (MnR); and pink, ventral tegmental area (VTA). Fibre tracts in the control, but not in the patient with coma, connect with the intralaminar nuclei (central lateral nuclei $(\mathrm{CL})$ and centromedian/parafascicular complex (CEM/Pf)) and the reticular nuclei (Ret) of the thalamus. Two midbrain haemorrhages (Haem) in the patient with coma are rendered in red. d

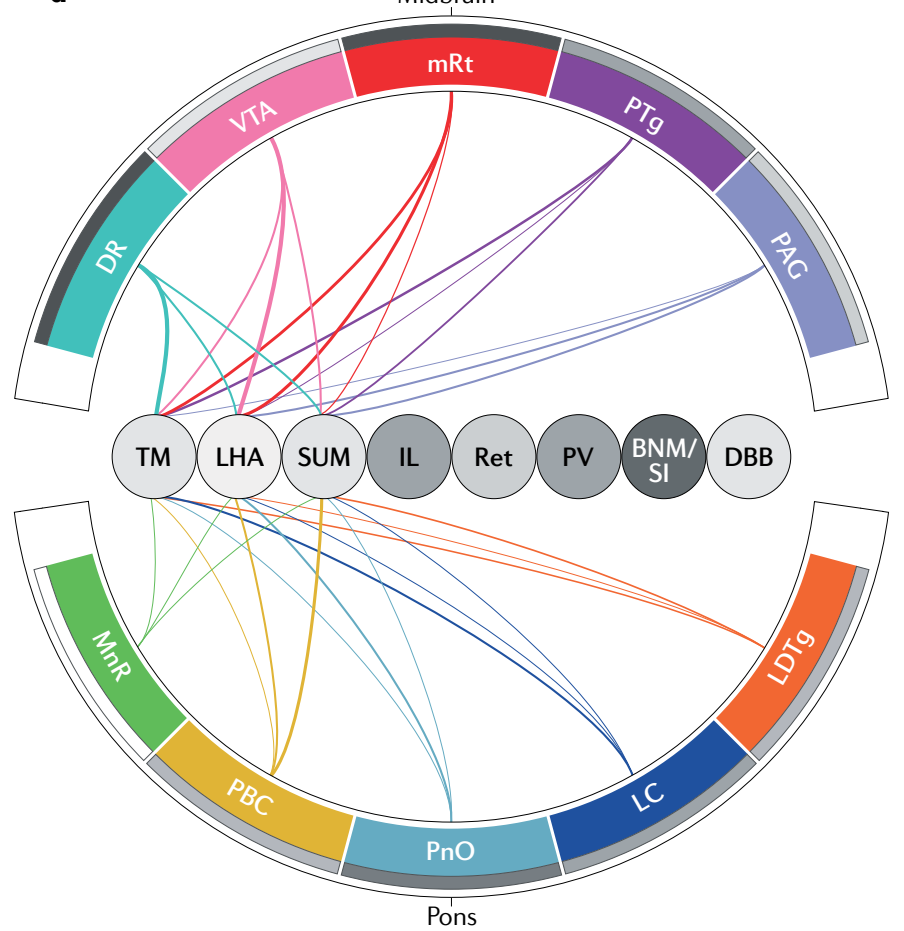

Nodal injury

$0 \%$

Connectivity probability

0.01

1.00

$\mathbf{c , d} \mid$ Ascending arousal network connectograms for the control (part c) and the patient with coma (part d). Brainstem nuclei are listed on the outside of the circle, and their subcortical targets are shown on the inside of the circle. Lines indicate connections between network nodes, with line thickness being proportional to the number of tracts visualized. Brainstem connections with thalamic and basal forebrain nuclei were disrupted in the patient with coma (part d), but connections with the hypothalamus were partially preserved. BNM/SI, nucleus basalis of Meynert/substantia innominata; DBB, diagonal band of Broca; IL, intralaminar nuclei of thalamus; LDTg, laterodorsal tegmental nucleus; LHA, lateral hypothalamic area; $\mathrm{mRt}$, mesencephalic reticular formation; PAG, periaqueductal grey; $\mathrm{PnO}$, pontis oralis; PV, paraventricular nucleus of the thalamus; SUM, supramammillary nucleus of the hypothalamus; TM, tuberomammillary nucleus of the hypothalamus. Parts $\mathbf{a}$ and $\mathbf{b}$ reprinted with permission from REF. ${ }^{109}$, OUP. 
achieve good outcomes, including return to a home environment and some level of independence ${ }^{89,151}$. Furthermore, evidence from one study indicates that particular EEG patterns in patients with MSE can predict outcomes ${ }^{152}$. Patients with MSE and a continuous background with narrow vertex discharges in lockstep with myoclonic jerks have a better chance of recovery (and better response to anti-seizure drugs) than those with MSE and a suppressed or suppression-burst EEG pattern with high-amplitude polyspikes, indicating that the background EEG rhythm is the most important EEG measure for predicting recovery ${ }^{152}$.
Evoked potentials are a measure of the time-locked EEG response to an external somatosensory, visual or auditory stimulus. Evoked potentials reflect early responses to stimulation - the first $\sim 100 \mathrm{~ms}$ - whereas ERPs reflect subsequent information processing and the cognitive aspects of the brain's response to stimulation ${ }^{153}$. Evoked potentials can be used to assess the integrity of specific sensory pathways, that is, the transmission of tactile sensation to the somatosensory cortex, providing reliable information about the overall impact of diffuse brain injury. For example, measurement of somatosensory evoked potentials are frequently used to aid a Lesion mapping

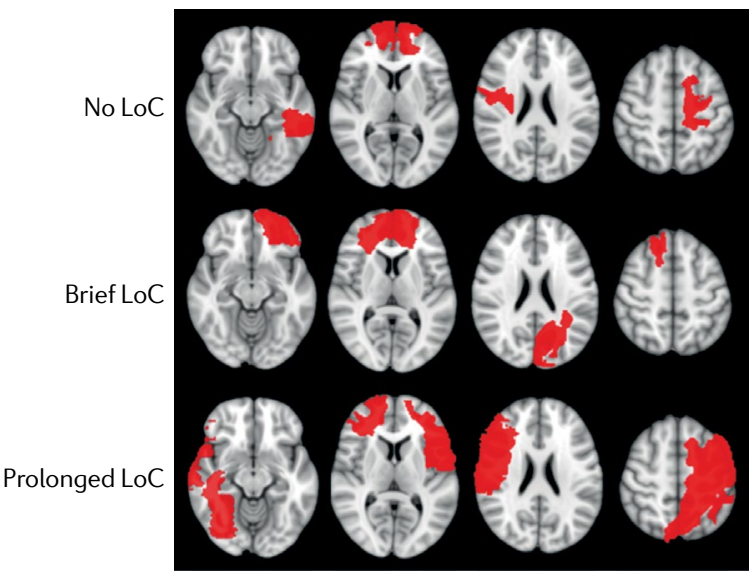

b Lesion connectivity

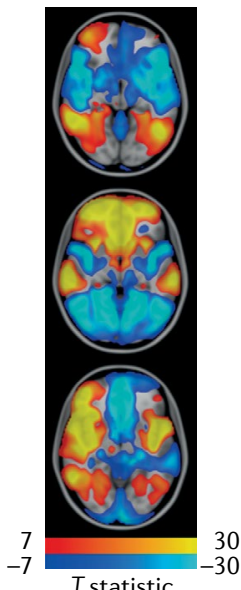

C Association with LoC 0.005

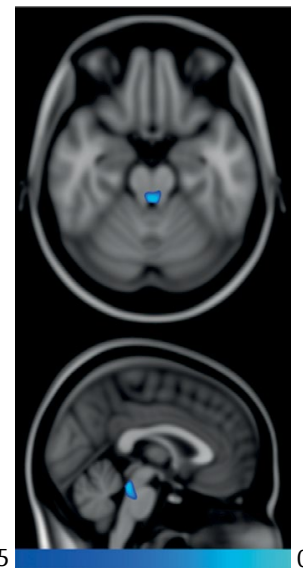

$P$ value

d Network identification

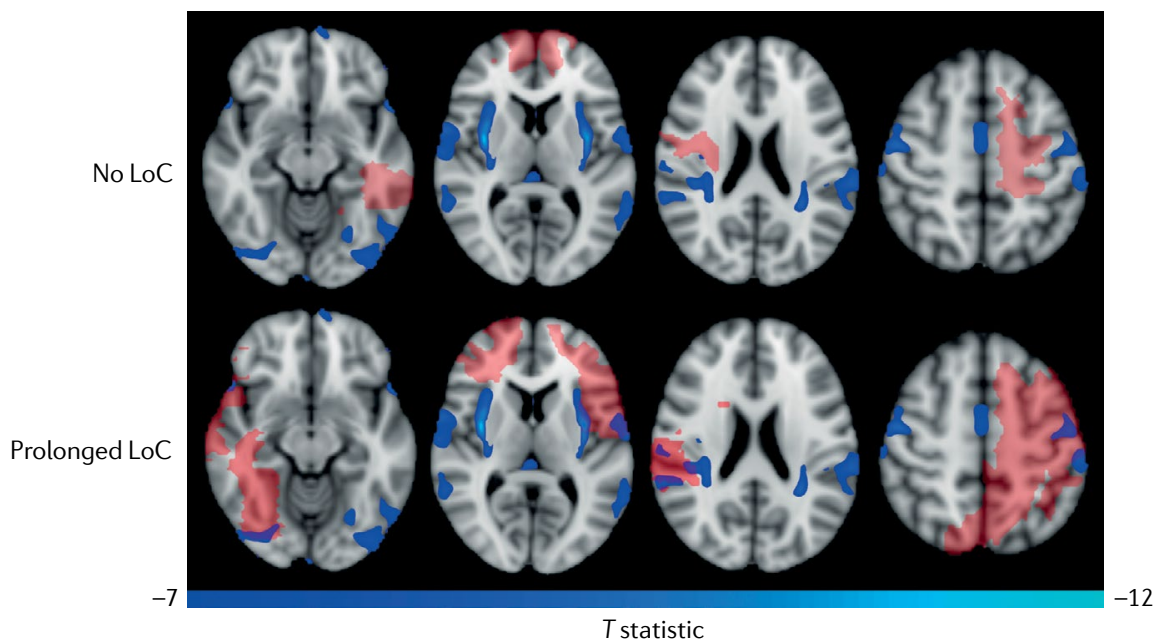

Fig. 3 | Mapping loss of consciousness to a common brain network using the human connectome. A novel technique termed 'lesion network mapping' was used to test whether lesions producing prolonged loss of consciousness (LoC) map to a distributed brain network or a single brain region. a | A sample of 171 individuals who experienced penetrating traumatic brain injuries and underwent head CT was classified into three groups: no LoC, brief ( $<1$ day) LoC and prolonged ( $>1$ day) LoC. Lesions were transformed onto a common brain template. Representative lesions from three individuals are shown in red. $\mathbf{b} \mid$ The brain regions functionally connected to each lesion location were identified using a database of 1,000 resting state functional MRI scans from healthy controls. c | Using voxel-wise ordinal logistic regression, the investigators determined that a lesion's connectivity with a specific region of the brainstem tegmentum (blue) was the strongest predictor of LoC. $\mathbf{d}$ |The network of cortical regions functionally connected to the identified brainstem region (blue) visualized alongside representative lesions (semi-transparent red). The lesions that caused no LoC show no overlap with the functional connectivity map whereas the lesions that caused prolonged LoC do overlap with the functional connectivity map. These findings suggest that lesions causing prolonged LoC injure a widely distributed brain network that is functionally connected to the brainstem tegmentum, known to contain consciousness-promoting arousal nuclei ${ }^{289}$. Adapted from REF. ${ }^{133}$, CC BY 4.0 (https://creativecommons.org/licenses/by/4.0/). 
prognostication following cardiac arrest ${ }^{154}$ and $\mathrm{TBI}^{155}-$ the absence of a negative potential $20 \mathrm{~ms}$ after peripheral stimulation reflects loss of thalamocortical integrity.

Multiple EEG features, including a well-organized EEG background and EEG reactivity to sensory stimulation, correlate with the results of behavioural assessments of consciousness ${ }^{156,157}$. These EEG features indicate preserved thalamocortical integrity, as corroborated by fMRI and fluorodeoxyglucose (FDG) PET data ${ }^{156}$. Re-emergence of these EEG features correlates with recovery of consciousness and can precede behavioural recovery ${ }^{158}$. Importantly, qualitative assessment of the EEG background and EEG reactivity for prognostication is feasible in a clinical setting and is performed routinely at many institutions caring for patients with acute DoC.

Emerging evidence suggests that detection and prediction of recovery of consciousness can be enhanced by quantitative analysis of the spatial and temporal characteristics of the resting EEG signal, including power, frequency, correlation between electrodes and EEC complexity ${ }^{57,159,160}$. For example, experimental and clinical data suggest that the EEG spectrogram reflects the degree of thalamocortical disconnection underlying impairment of consciousness ${ }^{27,47,56}$. Other quantitative EEG measures include information-sharing approaches that measure functional connectivity, also known as coherence, between brain regions ${ }^{57,160}$. These measures have mostly been explored in patients with chronic DoC (discussed below), but preliminary evidence suggests that they provide similar diagnostic and prognostic utility in acute DoC. For example, in a case series that included patients with acute TBI, higher EEG complexity, also known as EEG entropy, was observed in MCS than in VS/UWS ${ }^{161}$. Levels of consciousness in patients with acute SAH and cardiac arrest also correlated with spectral power, supporting the mesocircuit hypothesis ${ }^{56,57}$. EEG spectral measures have been found to correlate with functional connectivity measures derived from resting-state $\mathrm{fMRI}^{162}$, supporting the network-based paradigm of acute DoC pathogenesis. However, the temporal progression of EEG changes in relation to behavioural changes is not fully understood. In the days following acute brain injury, confounders such as metabolic derangements (for example, renal or hepatic failure) and medications (for example, sedatives) can affect both behavioural and electrophysiological assessments ${ }^{15}$. Advanced analytical techniques that account for these ICU confounders of EEG patterns in patients with acute DoC are actively being pursued ${ }^{54}$.

Chemical biomarkers. Although beyond the scope of this Review, chemical biomarkers are an integral component of the assessment of neuronal injury in patients with acute DoC, most importantly in patients with cardiac arrest. The most commonly studied chemical biomarker is neuron-specific enolase, the measurement of which is confounded by its presence in red blood cells, platelets and some neuroendocrine and pancreatic tumours ${ }^{163}$. Newer biomarkers, including serum Tau ${ }^{164}$ and neurofilament light chain ${ }^{165}$, have shown a higher specificity for brain injury post cardiac arrest, and are the subject of ongoing studies.

\section{Cognitive motor dissociation}

Some patients retain capacity for sentience and volitional thought without self-expression or motoric output, particularly in the ICU setting with endotracheal intubation, sedation, pain, injury to motor pathways and comorbid medical illness. This phenomenon, which was first demonstrated in the chronic setting using task-based fMRI ${ }^{166}$, has been described using various terms, including "functional locked-in syndrome", "covert cognition"167, "non-behavioural MCS $\left(\mathrm{MCS}^{\star}\right)$ "168 and "cognitive motor dissociation (CMD)" ${ }^{11}$. We prefer the term CMD, as it conveys a graded and hierarchical, rather than a binary, dissociation between cognition and motoric output.

The potential to detect CMD in patients with acute DoC is a major motivator for implementing task-based fMRI and EEG techniques in the ICU (FIG. 4). In a task-based fMRI or EEG study, a participant is instructed to follow a command by performing a motor imagery ("imagine opening and closing your hand"), spatial navigation ("imagine walking through the rooms of your house") or motor action ("open and close your hand") task ${ }^{28}$. During the task, volitional brain activity is measured and compared with resting brain activity, which is typically measured after an instruction to stop performing the task. From 2017 onwards, several studies using task-based fMRI and EEG paradigms identified CMD in some ICU patients who seemed unresponsive on behavioural examination ${ }^{95,169,170}$ (FICS 4 and 5). The precise prevalence of CMD in patients with acute $\mathrm{DoC}$ is unknown, as only a small number of studies have tested for CMD in the $\mathrm{ICU}^{95,169,170}$. In the largest study of CMD in the ICU to date, task-based EEG was performed at a single centre in 104 patients with acute DoC caused by traumatic and non-traumatic aetiologies ${ }^{95}$. The frequency of CMD detection was $15 \%(n=16)$, providing preliminary evidence that the rate of CMD in the ICU is similar to the $15-20 \%$ rate reported by a meta-analysis in patients with subacute-to-chronic $\mathrm{DoC}^{171}$, as discussed below. Moreover, this ICU study found that patients with acute CMD have a higher likelihood of functional recovery at 1 year post injury, as measured by the Glasgow Outcome Scale - Extended, than patients without acute CMD (odds ratio $=4.6$ (95\% confidence interval $1.2-17.1))^{95}$. This finding suggests that covert consciousness, that is, $\mathrm{CMD}$, has a similar prognostic relevance to overt consciousness in ICU patients with acute brain injuries.

Evidence indicates that task-based fMRI or EEG studies have a high rate of false negative findings, which is important to consider when interpreting the results. For example, some ICU patients who show behavioural evidence of command-following, that is, individuals with a diagnosis of MCS+ or confusional state, are unable to perform a motor imagery task ${ }^{169,170}$, possibly owing to confounding by sedation, head motion or inattention. Furthermore, the results of task-based motor imagery fMRI and EEG studies of healthy individuals have suggested that the false negative rate of this approach can be as high as $\sim 25 \%{ }^{169,172}$. Thus, absence of task-based motor imagery fMRI responses does not prove that a patient is incapable of command-following and does not necessarily connote a poor prognosis. Task-based 


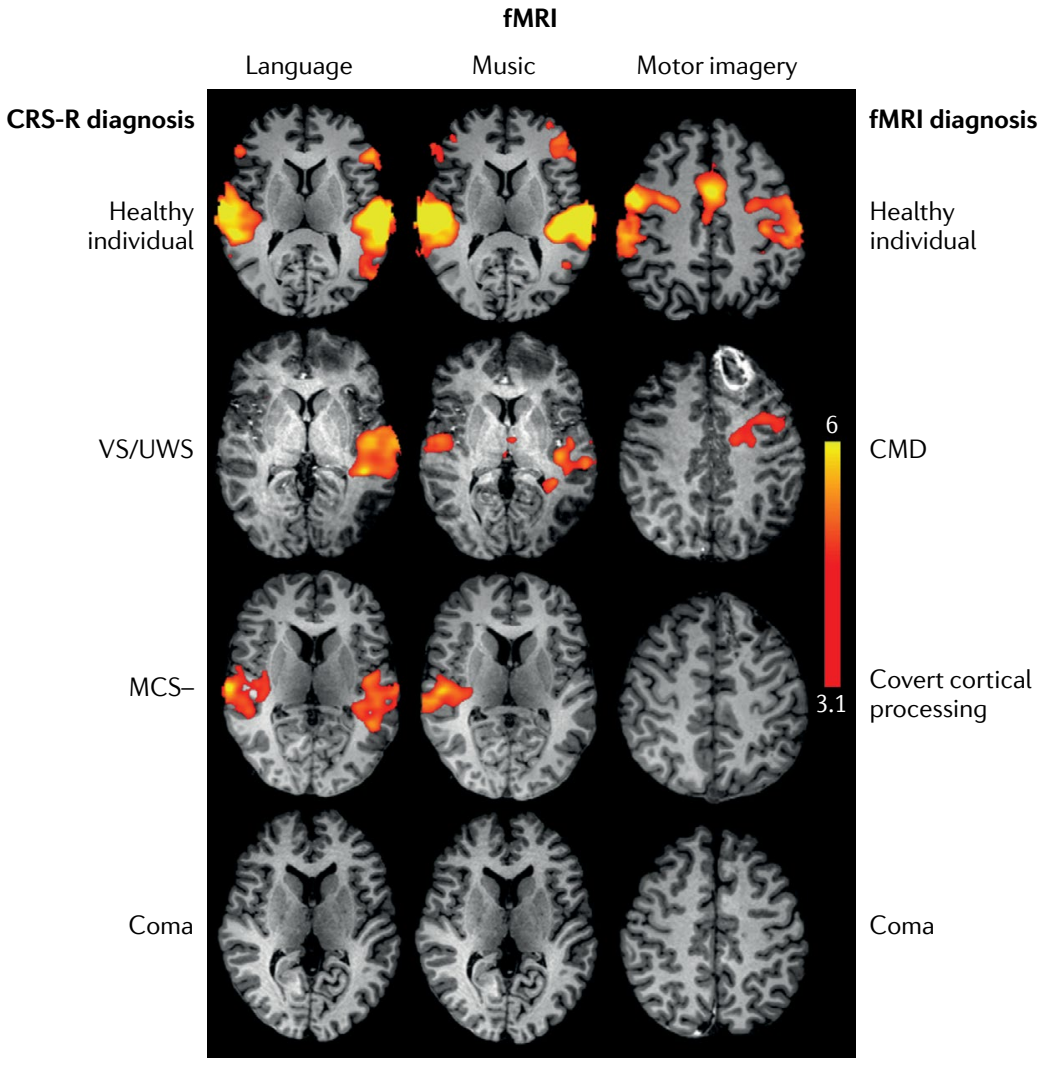

Fig. 4 | Task-based functional MRI detects cognitive motor dissociation in the intensive care unit. Functional MRI (fMRI) results for language, music and motor imagery paradigms for a healthy individual and patients with a broad range of behavioural diagnoses after acute severe traumatic brain injury. The second row shows scans from a patient with a behavioural diagnosis of vegetative state/unresponsive wakefulness syndrome (VS/UWS) but fMRI evidence of command-following, resulting in an fMRI diagnosis of cognitive motor dissociation (CMD). The third row shows scans from a patient with a behavioural diagnosis of minimally conscious state without language (MCS-) but $\mathrm{fMRI}$ evidence of association cortex activation to language and music stimuli, resulting in an fMRI diagnosis of covert cortical processing. The patients diagnosed with CMD, covert cortical processing and coma all recovered consciousness, communication and functional independence, highlighting the prognostic limitations and confounders associated with $\mathrm{fMRI}$ in the intensive care unit (for example, the comatose patient was on a propofol drip during the scan). Thus, a negative $\mathrm{fMRI}$ result should not be interpreted as a reliable indicator of poor outcome. fMRI data are shown as Z-statistic images thresholded at cluster-corrected Z scores of 3.1 (inset colour bar) and superimposed upon T1-weighted axial images. CRS-R, Coma Recovery Scale — Revised. Reprinted with permission from REF. ${ }^{169}$, OUP. or the ability to use an assistive communication device. When choosing between task-based fMRI or EEG for the detection of CMD in behaviourally unresponsive patients, logistics such as patient transport, the ability for repeat testing and spatial representation of the signal in relation to the injury have to be taken into account. Major advantages of task-based EEG include repeatability and employability at the bedside, which mitigate logistical challenges and eliminate the need for transport. However, task-based fMRI can provide more neuroanatomic specificity about covert brain responses. For both approaches, care is required when designing the task paradigm (we recommend a block design) and planning the statistical analysis, as errors might lead to a false positive diagnosis of $\mathrm{CMD}^{172,174}$. Reproducibility and the impact of confounders, such as sedation, metabolic derangement, artefacts and seizures, should also be considered when interpreting the results. Approaches that integrate task-based fMRI and EEG are currently being used to characterize the clinical characteristics of CMD in patients in the ICU and provide insights into its underlying mechanisms $s^{169}$. Meaningful communication with CMD patients is currently infeasible but, in the future, EEG-supported brain-computer interface systems could enable patients with acute CMD to communicate ${ }^{175,176}$.

fMRI and EEG approaches that use language and music as a stimulus to elicit brain activation have provided additional insights into preservation of cortical function in patients in the ICU with acute DoC, but the prognostic and therapeutic relevance of these approaches is unclear. Importantly, the passive response of the brain to a stimulus is not considered definitive evidence of consciousness ${ }^{177,178}$. Instead, only active, volitional modulation of cortical activity in response to a task indicates command-following and, hence, consciousness ${ }^{11}$. Nevertheless, association cortex responses to language and music in patients who do not show evidence of language function on behavioural examination - a phenomenon for which we propose the term 'covert cortical processing ${ }^{169,177-181}$ (FIG. 1) - might provide additional information about preserved neural networks that could support cognitive and functional recovery ${ }^{179}$.

With ongoing advances in fMRI and EEG techniques, and as experience with stimulus-based and task-based fMRI and EEG in the ICU grows, it is increasingly likely that these investigational techniques will be used clinically to detect and predict recovery of consciousness in patients with acute DoC. Indeed, the 2020 European Academy of Neurology guideline for the management of patients with DoC recommends that task-based fMRI and EEG be performed "whenever feasible ... to complement behavioural assessment in patients without command following at the bedside" ${ }^{182}$. This guideline also proposes that patients with DoC should be classified according to the highest level of consciousness revealed by behavioural, fMRI or EEG testing - an approach that is also being advocated by the DoC research community ${ }^{183,184}$.

\section{Treatments}

The first goal of treatment of patients with acute DoC is to identify causes of altered consciousness that can be rapidly corrected ${ }^{15}$. For example, acute hydrocephalus 
can be treated with cerebrospinal fluid diversion via an external ventricular drain, leading to rapid restoration of consciousness in some patients ${ }^{15}$. Similarly, correction of toxic, metabolic or endocrinologic derangements such as opioid overdose, hyponatraemia or

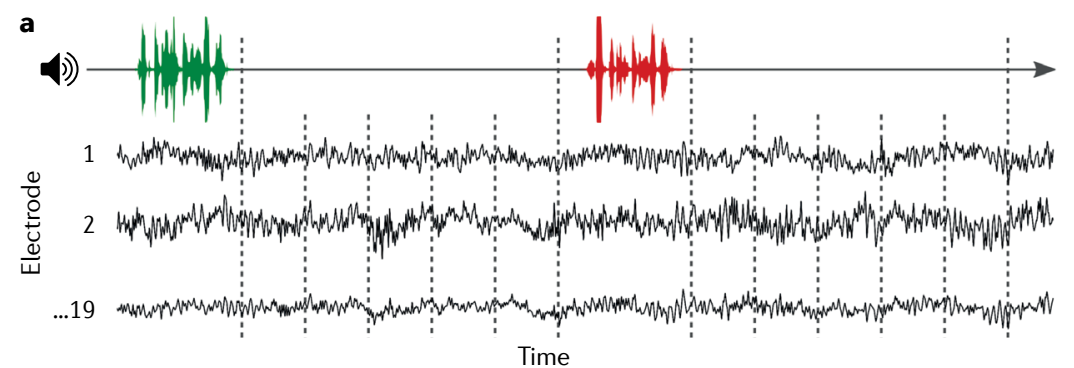

b
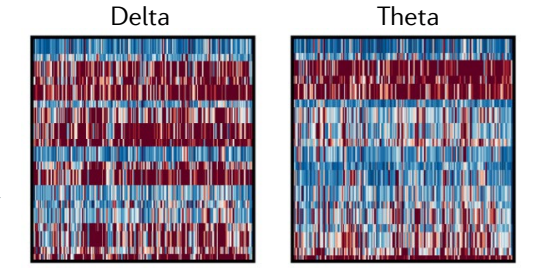

Alpha
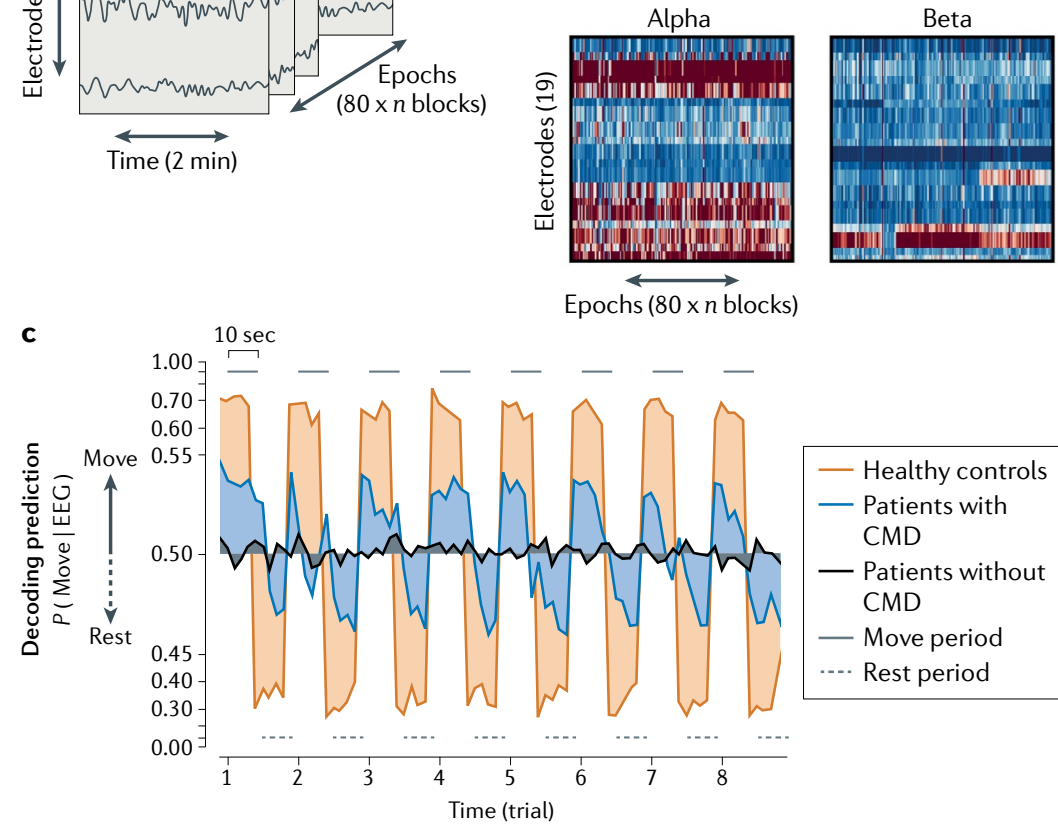

Fig. 5 | EEG detection of cognitive motor dissociation in the intensive care unit predicts 1-year functional recovery. Task-based EEG data were acquired in a sample of 104 patients with severe brain injuries of various aetiologies in the intensive care unit. a | EEG was recorded for $10 \mathrm{~s}$ following alternating instructions of "keep opening and closing your right hand" (green) and "stop opening and closing your right hand" (red). b | Power spectral density (PSD) analysis was applied to the EEG recorded from each electrode in four frequency bands: delta $(1-3 \mathrm{~Hz})$, theta $(4-7 \mathrm{~Hz})$, alpha $(8-13 \mathrm{~Hz})$ and beta $(14-30 \mathrm{~Hz}) . \mathbf{c} \mid$ Resulting features were used to train and test a machine learning algorithm (support vector machine). Classification performance for a given recording was assessed as the area under the receiver-operating characteristic curve. Decoding prediction is represented on the $y$ axis, reflecting the EEG response corresponding to a "keep moving" or a "stop moving" instruction. Lines represent group averages of the decoding prediction curves of healthy volunteers and patients with or without cognitive motor dissociation (CMD). This study found that intensive care unit patients with acute CMD had a higher likelihood of functional recovery at 1 year post injury than patients without CMD. Reprinted from REF. ${ }^{95}$, Claassen, J. et al. Detection of brain activation in unresponsive patients with acute brain injury. 380, 2497-2505. Copyright $\odot$ (2019) Massachusetts Medical Society. Reprinted with permission. hypothyroidism, respectively, can quickly restore consciousness. For patients whose aetiology of acute DoC is not rapidly reversible, neuroprotective therapies are often used to improve cerebral oxygenation or reduce metabolic demand. Neuroprotective reperfusion therapy is commonly used in patients with cerebral vasospasm caused by aneurysmal SAH ${ }^{185}$. Medical approaches, for example, blood pressure augmentation, and endovascular therapies are both used to restore perfusion to consciousness-promoting brain regions ${ }^{186}$. Targeted temperature management has been associated with improved outcomes in patients with acute DoC caused by cardiac arrest ${ }^{187-189}$. However, three large randomized control trials failed to demonstrate a benefit of targeted temperature management in patients with acute DoC following severe $\mathrm{TBI}^{190-192}$. Nevertheless, in our opinion, targeted temperature management can be considered in selected TBI patients with refractory intracranial hypertension ${ }^{193}$. Evidence indicates that decompressive hemicraniectomy also reduces mortality in this patient population $^{194}$.

Whereas neuroprotective therapies aim to prevent secondary neuronal injury, stimulant therapies aim to promote and accelerate restoration of neuronal function, and are now being tested in patients with acute $\mathrm{DoC}^{124,195,196}$. Initial results suggest that amantadine is well-tolerated in patients in the ICU with acute $\mathrm{DoC}^{197}$, but evidence for efficacy is lacking ${ }^{195,196}$. Reproducible EEG measures, that is, the hierarchical ABCD model, might detect changes in cerebral cortical function prior to clinical changes in patients with acute DoC treated with amantadine and could serve as early biomarkers to measure treatment effects in future clinical trials ${ }^{198}$. A potential association between early stimulant therapy and risk of seizures or excitotoxicity in patients with acute DoC remains a hypothetical concern, but is not supported by current evidence.

Device-related neuromodulation in the ICU is also gaining attention because of a recent first-in-human report of low-intensity focused ultrasound pulsation for central thalamic stimulation in a patient with acute $\mathrm{DoC}^{199}$. A barrier to developing targeted, consciousness-promoting pharmacological and device-related therapies for use in the ICU has been a lack of biomarkers that predict therapeutic responses. The network-based paradigm of recovery of consciousness has inspired efforts to map brain networks and identify connectomes amenable to neuromodulation in patients with acute $\mathrm{DoC}^{124}$.

\section{Subacute-to-chronic DoC \\ Detection and prediction of recovery}

Clinical examination. The CRS-R is the most widely used behavioural assessment tool in clinical care and research investigations, receiving the strongest recommendation from the American Congress of Rehabilitation Medicine task force on the basis of its psychometric properties for detecting consciousness in patients with subacute-to-chronic $\mathrm{DoC}^{200}$. The behavioural assessment of patients with subacute-to-chronic DoC was extensively reviewed in 2014 (REF. ${ }^{1}$ ). However, important updates to the behavioural assessment 
since 2014 include a refined approach to distinguishing MCS- from MCS+ and recent evidence that patients with MCS+ have lower levels of disability than patients with MCS- at the time of discharge from inpatient rehabilitation ${ }^{9}$. Additionally, the importance of performing multiple CRS-R examinations to optimize detection of volitional behaviours has now been demonstrated ${ }^{201}$. A new behavioural assessment tool, the Motor Behaviour Tool - Revised (MBT-r) ${ }^{202}$, has also been developed to complement the CRS-R by identifying subtle motor behaviours that reveal residual cognition. Evidence from a sample of 141 patients receiving inpatient rehabilitative care for severe brain injuries suggests that the MBT-r used in conjunction with the CRS-R detects consciousness with a greater sensitivity than the CRS-R alone ${ }^{203}$. Moreover, behavioural diagnoses derived from the MBT-r predicted functional recovery at discharge from the inpatient rehabilitation centre ${ }^{203}$. Finally, reports of 'late' recovery of consciousness, that is, recovery $>1$ year after injury ${ }^{204}$, have led new DoC guidelines to abandon the term 'permanent' when describing patients with VS/UWS 2 , 182,205 .

Imaging. The application of advanced structural and functional imaging during the subacute-to-chronic stage of DoC is motivated by similar diagnostic and prognostic goals to those in the acute setting, with a key difference being that imaging during the subacute-to-chronic stage is typically more feasible from a safety and logistical standpoint because the patients are not critically ill. For this reason, far more imaging studies have been performed in the subacute-to-chronic stage of recovery than in the acute stage. The foundational studies that first linked brain network dysfunction to subacute-to-chronic DoC used PET and identified metabolic dysfunction within multiple neural network nodes ${ }^{28}$. PET studies also revealed that patients with VS/UWS have altered connectivity between the posterior cingulate and frontal association cortices ${ }^{206}$, as well as between the thalamus, prefrontal and anterior cingulate cortices $^{207}$. Whether at rest or performing a task, patients with DoC demonstrated disruptions in functional networks essential for processing intrinsic thoughts and extrinsic stimuli ${ }^{136}$. Furthermore, PET provided the first evidence of neuronal responses within association cortices in patients who seemed to be unresponsive ${ }^{177,208}$, providing a conceptual foundation for subsequent studies of covert cortical processing.

As the field of advanced imaging matured, subsequent PET and $\mathrm{fMRI}$ studies revealed the functional dynamics of disrupted networks in individuals with DoC. A central insight was the observation that DMN connectivity might be a fundamental property of human consciousness. For example, multiple studies have identified DMN disconnections in patients with subacute-to-chronic DoC and cognitive dysfunction ${ }^{30,209-214}$, and DMN connectivity increases longitudinally as patients gradually recover consciousness ${ }^{36,210}$. Evidence indicates that DMN functional connectivity differentiates between MCS and VS/UWS with an accuracy of $>80 \%{ }^{215,216}$. Furthermore, $\mathrm{DMN}$ functional connectivity has been shown to predict recovery of consciousness $s^{31,217,218}$ and neurocognitive task performance ${ }^{209,211,212}$ across the time spectrum of recovery. Multimodal MRI studies have demonstrated that functional connectivity measurements in DoC correlate with the structural network architecture 209,211 . Indeed, the results of a diffusion tractography study supported a role for DMN structural connectivity in subacute-to-chronic DoC ${ }^{219}$. Collectively, these complementary structural-functional connectivity studies suggest that DMN connectivity is a diagnostic biomarker of consciousness and a potential predictor of ongoing recovery in the subacute-to-chronic stage of DoC.

Despite these encouraging findings, DMN connectivity alone cannot classify states of consciousness, as patients with subacute-to-chronic DoC show disruptions within additional functional networks, including networks involved in processing sensory stimuli and performing cognitive tasks, such as the auditory, visual, sensorimotor, salience and executive control networks ${ }^{215,217}$. The cognitive task networks are referred to as 'task-positive' or 'extrinsic' networks because they are most active during goal-directed tasks ${ }^{220}$. During resting wakefulness, extrinsic networks are functionally connected and are negatively correlated (anti-correlated) with 'intrinsic' resting networks such as the $\mathrm{DMN}^{221,222}$. Evidence from a longitudinal resting-state fMRI study of patients with severe TBI suggests that the re-emergence of anti-correlations between intrinsic and extrinsic networks contributes to recovery of consciousness ${ }^{36}$. The results of a recent multicentre resting-state fMRI study further supported the importance of internetwork interactions for consciousness by showing that dynamic patterns of correlated and anti-correlated network activity distinguish patients with MCS from patients with VS/UWS ${ }^{223}$.

Owing to methodological challenges, the contributions of subcortical networks to recovery of consciousness are poorly understood. For example, arousal nuclei in the brainstem are difficult to image because of skull base susceptibility artefacts and the pulsation artefacts from nearby blood vessels ${ }^{224}$. Furthermore, blood oxygen level-dependent fMRI signals within small brainstem nuclei have low signal to noise ratios; thus, ultra high-resolution $\mathrm{fMRI}$ sequences are required to accurately image these nuclei ${ }^{225}$. State of the art fMRI techniques are being developed with the aim of mapping the functional connectivity of subcortical networks ${ }^{225-227}$, and these techniques have the potential to yield new insights into mechanisms of recovery related to subcortical-cortical network reintegration.

Electrophysiology. Major progress has been made in the development of electrophysiological biomarkers of subacute-to-chronic DoC using resting and stimulus-based EEG approaches ${ }^{159,228}$. For example, a study in 2018 found electrophysiological evidence of sleep-like cortical off-periods in patients with VS/UWS, a finding observed in healthy sleeping individuals but not in healthy awake individual ${ }^{229}$. Evidence suggests that the presence of resting EEG features such as sleep architecture - in particular, synchronization of sleep spindles correlates with local changes in metabolism (measured using FDG PET) and fMRI network structure ${ }^{134}$, and can predict long-term recovery of consciousness in patients 
with subacute-to-chronic TBI or cardiac arrest ${ }^{230,231}$. These resting EEG features can be readily detected in the clinical setting. Resting EEG is also being used in the investigational setting to provide information about brain connectivity, and these EEG-based connectivity measures have been found to correlate with MRI connectivity measures and behavioural end points in patients with subacute-to-chronic $\mathrm{DoC}^{160,232}$. Moreover, several studies used resting EEG features to quantify the complexity of the recorded electrophysiological signal in patients with subacute-to-chronic DoC and found that complexity measures correlated with concomitant behavioural assessments, suggesting that this technique could be used to improve the accuracy of predicting recovery of consciousness ${ }^{159,160}$.

Electrophysiological recordings following somatosensory, auditory or visual stimulation have long been explored in research into subacute-to-chronic DoC. Inspired by studies in neuropsychology, these recordings consist of positive or negative EEG waveforms that are strongly time-locked to the stimulus and revealed by averaging repeated stimulations ${ }^{233}$. Studies of DoC have focused on waveforms that occur $>100 \mathrm{~ms}$ after stimulation, for example, the P300 wave or the N400 wave, and are thought to reflect higher-order processing of the stimulus $^{234,235}$. Initial evidence suggested that the presence of late-evoked potentials such as the P300 wave can predict recovery in patients with subacute-to-chronic DoC of a broad range of aetiologies ${ }^{236,237}$. However, evidence from a more recent study in 92 patients with subacute-to-chronic VS/UWS and MCS suggests that the $\mathrm{N} 400$ response, much more than the $\mathrm{P} 300$ response, predicts long-term recovery of consciousness and communication $^{238}$. Moreover, evidence indicates that the presence of the $\mathrm{P} 300$ response alone might not consistently distinguish between patients in VS/UWS and MCS $^{160}$.

Another electrophysiological measurement that enables the probing of brain responses to stimuli in patients with DoC is mismatch negativity, which is an ERP that occurs in response to an 'oddball' sound and is thought to be a pre-attentive or preconscious measure ${ }^{239}$. During measurement of mismatch negativity, a patient listens to a sequence of frequent, repetitive sounds that are interspersed with infrequent 'oddball' sounds that differ from the rest of the sequence, for example, in their pitch. Initial evidence suggests that the presence of mismatch negativity predicts clinical improvement, including a transition from VS/UWS to MCS, in patients with subacute-to-chronic DoC of traumatic and non-traumatic aetiologies ${ }^{235,240}$. Probing brain responses to multiple layers of regularities within an auditory stimulus is also being studied, as the resulting ERP might distinguish conscious versus subconscious processing ${ }^{153}$. These multiple layers of regularities consist of identical or deviant sounds within a sequence of five tones (local regularity or deviance) and between several blocks of five tones (global regularity or deviance). This analysis dissociates a local from a global response, but limited data exist regarding the feasibility and applicability of this approach in clinical practice ${ }^{241}$. An approach that quantifies cognitive modulation of the autonomic nervous system by measuring auditory ERP-related variations in heart rate has been explored in patients with DoC and shows some promise ${ }^{242}$. The ability of the brain to integrate and process information can also be tested in patients with subacute-to-chronic DoC by measuring the response to natural speech or music ${ }^{183,243}$. Analysing the natural speech envelope of the EEG recorded during speech might allow identification of patients with severe brain injury who demonstrate preserved cognitive function $^{183}$, such as covert cortical processing or CMD.

An alternative electrophysiological approach to probing brain networks in patients with subacute-to-chronic DoC is TMS-EEG, in which a targeted TMS pulse is administered to a patient who is being monitored with high-density EEG ${ }^{24,245}$. This TMS-EEG method directly assesses brain function by bypassing sensory system inputs and enables the calculation of a perturbation complexity index ${ }^{244,245}$, which represents the complexity of the cortical response, as reflected in the electrical signal recorded by EEG following TMS. Major advantages of the TMS-EEG approach are that it provides a single numerical value that strongly correlates with a patient's level of consciousness, and its methodological principles are firmly rooted in the integrated information theory of consciousness $^{246}$.

A future goal of this field of work will be to determine which electrophysiological techniques provide the greatest prognostic utility and mechanistic insight into recovery of consciousness. Central to this effort will be the standardization of data acquisition, processing and analysis, which will enhance the reproducibility and generalizability of advanced electrophysiological measures $^{174,228}$. Efforts are underway to test cross-site reliability and identify minimum data requirements, for example, the number of electrodes ${ }^{159,247}$. To promote access to electrophysiological measures, investigators are increasingly sharing data-acquisition protocols and analytic code $\mathrm{e}^{95,248}$.

\section{Cognitive motor dissociation}

In parallel with discoveries about network-based mechanisms of recovery, rapid progress has been made in the development of advanced imaging and electrophysiological tools to detect CMD in patients with subacute-to-chronic DoC $172,228,249-251$. Since the first demonstration of CMD in 2006 (REF. ${ }^{166}$ ), the detection of CMD with task-based fMRI and EEG has been replicated using multiple motor imagery, spatial imagery, auditory and visual paradigms $\mathrm{s}^{28,172,228,249,252-254}$. Evidence from one study indicates that combining multiple task-based paradigms improves the CMD detection rate ${ }^{255}$. Although the precise prevalence of subacute-to-chronic CMD is unknown, initial evidence suggests that CMD is more common in patients with traumatic DoC than in patients with non-traumatic DoC ${ }^{250}$, and a meta-analysis suggested that the overall prevalence of CMD in patients with subacute-to-chronic DoC is $15-20 \%{ }^{171}$. Even if this prevalence statistic proves to be an overestimate (owing to publication bias), hundreds of thousands of individuals worldwide are living with chronic $\mathrm{DoC}^{256,257}$, suggesting that the number of patients with DoC worldwide who have unrecognized CMD (owing to a lack of 
diagnostic testing) is potentially in the thousands. In our view, this possibility provides an ethical imperative to develop, implement and generalize diagnostic tools to identify patients with CMD.

The potential translational impact of the emerging literature on CMD is perhaps best evidenced by the recent incorporation of task-based fMRI and EEG techniques into clinical guidelines for patients with DoC. Task-based fMRI is referred to as a test with potential clinical utility for patients with subacuteto-chronic DoC in the 2018 DoC guideline ${ }^{2,205}$ sponsored by the American Academy of Neurology, the American Congress of Rehabilitation Medicine and the US National Institute on Disability, Independent Living, and Rehabilitation Research. In addition, as mentioned previously, the 2020 European Academy of Neurology guideline ${ }^{182}$ recommends the use of taskbased fMRI, EEG and PET techniques for assessment of patients with acute and subacute-to-chronic DoC "whenever feasible". With respect to prognostic accuracy, a clinical validation study found FDG PET to be superior to $\mathrm{fMRI}$ in predicting long-term outcome in patients with subacute-to-chronic DoC ${ }^{250}$, underscoring the importance of a multimodal approach to CMD detection and prognostication ${ }^{258}$. FDG PET has a high sensitivity and specificity ( $94 \%$ ) for identifying consciousness (but not CMD per se) in patients with subacute-to-chronic $\mathrm{DoC}^{26}$; in contrast, although $\mathrm{fMRI}$ has a high specificity for CMD detection, it is insensitive ( $45 \%)$ to detecting consciousness in subacute-to-chronic DoC ${ }^{250}$.

As fMRI, EEG and PET begin to gain acceptance as clinically useful tests for detecting and predicting recovery of consciousness, clinicians will likely face new challenges regarding resource allocation and generalization of these advanced methods. One approach is to screen patients with subacute-to-chronic DoC for their potential to have CMD, an approach that recently showed promise in two studies that measured EEG responses to the natural speech envelope of spoken language ${ }^{183,259}$. These responses were associated with fMRI evidence of CMD, suggesting that EEG-based assessment of the natural speech envelope could be used as a screening tool to identify patients who warrant subsequent assessment for CMD with task-based fMRI or EEG.

\section{Treatments}

Most trials of pharmacological treatments in patients with subacute-to-chronic DoC have tested stimulants that promote dopamine signalling ${ }^{260}$, for example, amantadine ${ }^{261}$, methylphenidate ${ }^{262}$, levodopa ${ }^{263}$, bromocriptine $^{264}$ and subcutaneous apomorphine $e^{265}$. The electrophysiological interventions that have been tested as treatments for subacute-to-chronic DoC include deep brain stimulation ${ }^{40}$, TMS $^{266}$, transcranial direct current stimulation ${ }^{267}$, low-intensity focused ultrasound pulsation ${ }^{199}$ and vagal nerve stimulation ${ }^{268}$. A broad range of additional non-pharmacological therapies, including sensory stimulation ${ }^{269}$ and music therapy ${ }^{270}$, are also being tested. However, to date, the only therapy that has shown benefit in a randomized placebo-controlled trial is amantadine (TABLES 2 and 3), which was associated with accelerated functional recovery in patients with traumatic VS/UWS and MCS at 4-16 weeks post injury ${ }^{261}$. Hence, treatment with amantadine is recommended in the 2018 DoC guidelines ${ }^{2,205}$ for this patient population, with the aim of promoting recovery. In patients who do not respond to amantadine, we believe that the administration of other pharmacological stimulants, for example methylphenidate, on a trial basis is reasonable. As it carries a low risk of adverse effects, we also recommend that patients with subacute-to-chronic DoC resistant to amantadine receive zolpidem on a trial basis. Zolpidem is a GABA-ergic medication with a paradoxical awakening effect in $\sim 5 \%$ of patients with subacute-to-chronic $\mathrm{DoC}^{271}$.

As evidence indicates that some pharmacological and electrophysiological therapies can reactivate arousal pathways in selected patients with subacute and chronic brain injuries $^{261,265,272}$, biomarkers are urgently needed to identify patients whose brain networks might be amenable to therapeutic modulation ${ }^{273}$. Evidence from single-photon emission computed tomography ${ }^{274}$ and $\mathrm{PET}^{275}$ studies of dopaminergic neurotransmission suggests that alterations to dopaminergic signalling can be detected in vivo in patients with severe brain injuries. Dopaminergic tone is crucial to restoring firing rates within a deafferented striatum $^{275}$. Therefore, these neuroimaging techniques could be used to identify patients likely to respond to dopaminergic neuromodulatory agents.

Neuroprotective therapies that aim to reduce neuroinflammation and prevent neurodegeneration have also been developed for patients with subacute-to-chronic $\mathrm{DoC}^{276,277}$. Chronic neuroinflammation, as evidenced by the presence of histopathological ${ }^{278}$ and $\mathrm{PET}^{279}$ markers of microglial activity, has been observed years after severe TBI. However, in a clinical trial of the antibiotic minocycline, the aim of which was to reduce chronic inflammation, patients receiving the drug showed higher serological levels of a neurodegeneration marker, neurofilament light protein, than patients not receiving the drug $^{279}$. This unexpected observation suggests that chronic neuroinflammation can actually be beneficial and that downregulation of neuroinflammation can have unintended consequences. Several multisite studies have been launched with the aim of elucidating the pathophysiological mechanisms underlying posttraumatic neurodegeneration ${ }^{280-283}$, but effective neuroprotective therapies have yet to be developed.

Importantly, many patients who recover from $\mathrm{DoC}$ experience lifelong cognitive impairments ${ }^{62}$, possibly because the functional dynamic range of their neocortical, thalamic and striatal neurons is limited, as discussed above. In a recent study of central thalamic deep brain stimulation in a patient with longstanding (18-year) cognitive impairment following a severe TBI, broad increases in the neuropsychological functions linked to frontostriatal neurons, which control executive attention, vigilance and resistance to fatigue, were observed after 3 months of 12 -h daily stimulation ${ }^{284}$. As new therapies are applied across the time spectrum of recovery, imaging and electrophysiological biomarkers should become more precise, along with systematic evaluation of electrical and pharmacological stimulation strategies. Improving the lives of patients with $\mathrm{DoC}$ will also 
Table 2 | Key studies of pharmacological interventions for subacute-to-chronic disorders of consciousness

\begin{tabular}{|c|c|c|c|c|c|c|c|c|}
\hline Intervention & Study & $\begin{array}{l}\text { Number of } \\
\text { participants } \\
\text { and cause } \\
\text { of DoC }\end{array}$ & $\begin{array}{l}\text { Time since } \\
\text { injury }\end{array}$ & Diagnosis & Study design & Procedure & Results & Comments \\
\hline Apomorphine & $\begin{array}{l}\text { Fridman et al. } \\
(2010)^{265}\end{array}$ & $8 \mathrm{TBI}$ & 46-104 days & $\begin{array}{l}6 \mathrm{VS} / \\
\text { UWS, } \\
2 \mathrm{MCS}\end{array}$ & Open-label & $\begin{array}{l}\text { Subcutaneous } \\
\text { infusion } \\
\text { administered for } \\
12-16 \mathrm{~h} / \text { day at } \\
\text { dose of up } \\
\text { to } 8 \mathrm{mg} / \mathrm{h} \text { for } \\
84-180 \text { days }\end{array}$ & $\begin{array}{l}\text { All } 8 \text { participants } \\
\text { responded to } \\
\text { commands (onset } \\
1-62 \text { days after } \\
\text { initiation of } \\
\text { apomorphine) } \\
\text { and all had } \\
\text { improved DRS } \\
\text { scores at } 1 \text { year } \\
\text { after treatment } \\
\text { initiation; no } \\
\text { serious adverse } \\
\text { events related } \\
\text { to apomorphine }\end{array}$ & $\begin{array}{l}\text { No control } \\
\text { group; unable } \\
\text { to differentiate } \\
\text { drug response } \\
\text { from natural } \\
\text { recovery }\end{array}$ \\
\hline Zolpidem & $\begin{array}{l}\text { Whyte et al. } \\
(2014)^{271}\end{array}$ & $\begin{array}{l}84 \mathrm{TBI} \text { and } \\
\text { non-TBI }\end{array}$ & $>4$ months & $\begin{array}{l}18 \mathrm{VS} / \\
\text { UWS, } \\
66 \mathrm{MCS}\end{array}$ & $\begin{array}{l}\text { Double-blind, } \\
\text { crossover }\end{array}$ & $\begin{array}{l}\text { Single dose of } \\
10 \text { mg zolpidem } \\
\text { and, if } \\
\text { improvement, } \\
\text { a placebo- } \\
\text { controlled phase; } \\
\text { evaluations at } \\
\text { baseline and } 1 \mathrm{~h} \text {, } \\
2 \mathrm{~h} \text { and } 3 \mathrm{~h} \text { after } \\
\text { zolpidem or } \\
\text { placebo }\end{array}$ & $\begin{array}{l}4 \text { participants } \\
\text { showed }>5 \text {-point } \\
\text { increase in CRS-R } \\
\text { during treatment } \\
\text { with zolpidem; } \\
1 \text { participant } \\
\text { had severe } \\
\text { adverse effect } \\
\text { (agitation causing } \\
\text { tracheostomy } \\
\text { tube to dislodge) }\end{array}$ & $\begin{array}{l}\sim 5 \% \text { response } \\
\text { rate to } \\
\text { zolpidem }\end{array}$ \\
\hline
\end{tabular}

AAN, American Academy of Neurology; ACRM, American Congress of Rehabilitation Medicine; BID, twice per day; CRS-R, Coma Recovery Scale — Revised; DoC, disorders of consciousness; DRS, Disability Rating Scale; FDG, fluorodeoxyglucose; GCS, Glasgow Coma Scale; ICH, intracerebral haemorrhage; IVH, intraventricular haemorrhage; MCS, minimally conscious state; NIDILRR, National Institute on Disability, Independent Living, and Rehabilitation Research; SAH, subarachnoid haemorrhage; TBI, traumatic brain injury; VS/UWS, vegetative state/unresponsive wakefulness syndrome.

require advances in the usability of assistive communication devices, which thus far have proved challenging to deploy and have provided a means of self-expression for only a small number of patients ${ }^{134}$.

\section{Conclusions}

Some patients with DoC have the potential to recover consciousness, communication and functional independence. Recovery of consciousness can occur at any time across the temporal spectrum of DoC, from the acute to the subacute-to-chronic time periods. Imaging and electrophysiology are rapidly adding to our knowledge of the mechanisms of recovery and providing new opportunities for the detection of CMD in patients who seem unresponsive on behavioural examination. However, the brain networks and neuronal mechanisms that are essential for recovery of consciousness have yet to be fully elucidated. Furthermore, reliable prognostic tools 
Table 3 | Key studies of non-pharmacological interventions for subacute-to-chronic disorders of consciousness

\begin{tabular}{|c|c|c|c|c|c|c|c|c|}
\hline Intervention & Study & $\begin{array}{l}\text { Number of } \\
\text { participants } \\
\text { and cause } \\
\text { of DoC }\end{array}$ & $\begin{array}{l}\text { Time since } \\
\text { injury }\end{array}$ & Diagnosis & Study design & Procedure & Results & Comments \\
\hline $\begin{array}{l}\text { Deep brain } \\
\text { stimulation } \\
\text { (DBS) }\end{array}$ & $\begin{array}{l}\text { Schiff } \\
\text { et al. } \\
(2007)^{272}\end{array}$ & $1 \mathrm{TBI}$ & 6 years & $1 \mathrm{MCS}$ & $\begin{array}{l}\text { Single- } \\
\text { subject, } \\
\text { double- } \\
\text { blind, } \\
\text { alternating } \\
\text { crossover }\end{array}$ & $\begin{array}{l}\text { Bilateral } \\
\text { electrical DBS } \\
\text { of the central } \\
\text { thalamus; CRS-R } \\
\text { assessments } \\
\text { performed } \\
\text { during DBS 'on' } \\
\text { and 'off' periods }\end{array}$ & $\begin{array}{l}\text { Increased frequency } \\
\text { of cognitively } \\
\text { mediated behaviours, } \\
\text { functional limb } \\
\text { control and oral } \\
\text { feeding, during 'on' } \\
\text { periods }\end{array}$ & $\begin{array}{l}\text { Proof of } \\
\text { principle that } \\
\text { stimulation } \\
\text { of the central } \\
\text { thalamus } \\
\text { can lead to } \\
\text { cognitive and } \\
\text { functional gains } \\
\text { in patients with } \\
\text { chronic DoC }\end{array}$ \\
\hline $\begin{array}{l}\text { Transcranial } \\
\text { direct current } \\
\text { stimulation }\end{array}$ & $\begin{array}{l}\text { Thibaut } \\
\text { et al. } \\
(2014)^{267}\end{array}$ & $\begin{array}{l}30 \text { non-TBI, } \\
25 \mathrm{TBI}\end{array}$ & $\begin{array}{l}1 \text { week-19 } \\
\text { years }\end{array}$ & $\begin{array}{l}25 \mathrm{VS} / \\
\text { UWS, } \\
30 \mathrm{MCS}\end{array}$ & $\begin{array}{l}\text { Double- } \\
\text { blind, sham- } \\
\text { controlled, } \\
\text { crossover }\end{array}$ & $\begin{array}{l}\text { Single session } \\
\text { ( } 20 \text { min) of } \\
\text { stimulation } \\
\text { and sham over } \\
\text { the left DLPFC, } \\
\text { with CRS-R } \\
\text { before and after } \\
\text { stimulation }\end{array}$ & $\begin{array}{l}\text { In } 13 \text { participants } \\
\text { with MCS and } \\
2 \text { participants with } \\
\text { VS/UWS, the CRS-R } \\
\text { score was higher } \\
\text { after stimulation than } \\
\text { before stimulation; } \\
\text { no side effects }\end{array}$ & $\begin{array}{l}\text { Proof of } \\
\text { principle that } \\
\text { non-invasive } \\
\text { stimulation } \\
\text { of DLPFC } \\
\text { can lead to } \\
\text { cognitive } \\
\text { gains }\end{array}$ \\
\hline $\begin{array}{l}\text { Repetitive } \\
\text { transcranial } \\
\text { magnetic } \\
\text { stimulation }\end{array}$ & $\begin{array}{l}\text { Cincotta } \\
\text { et al. } \\
(2015)^{288}\end{array}$ & $\begin{array}{l}9 \text { non-TBI, } \\
2 \mathrm{TBI}\end{array}$ & 9-85 months & $\begin{array}{l}11 \mathrm{VS} / \\
\text { UWS }\end{array}$ & $\begin{array}{l}\text { Double- } \\
\text { blind, } \\
\text { crossover }\end{array}$ & $\begin{array}{l}\text { Five sessions } \\
\text { of } 20-\mathrm{Hz} \\
\text { stimulation or } \\
\text { sham for } 10 \mathrm{~min}, \\
\text { for a total of } \\
1,000 \text { pulses } \\
\text { delivered in } \\
5 \text { trains, over } \\
\text { the left primary } \\
\text { motor cortex for } \\
5 \text { consecutive } \\
\text { days; EEG } \\
\text { and CRS-R } \\
\text { performed } \\
\text { before and after } \\
\text { stimulation }\end{array}$ & $\begin{array}{l}\text { No change in } \\
\text { behavioural or EEG } \\
\text { measurements; } \\
\text { no side effects }\end{array}$ & $\begin{array}{l}\text { Small sample } \\
\text { size }\end{array}$ \\
\hline $\begin{array}{l}\text { Vagal nerve } \\
\text { stimulation }\end{array}$ & $\begin{array}{l}\text { Corazzol } \\
\text { et al. } \\
(2017)^{268}\end{array}$ & $1 \mathrm{TBI}$ & 15 years & 1 VS/UWS & $\begin{array}{l}\text { Single- } \\
\text { subject }\end{array}$ & $\begin{array}{l}\text { Stimulation } \\
\text { (up to } 1.5 \mathrm{~mA} \\
\text { intensity) } \\
\text { administered } \\
\text { over } 6 \text { months, } \\
\text { with CRS-R, } \\
\text { EEG and FDG } \\
\text { PET performed } \\
\text { before and after } \\
\text { stimulation }\end{array}$ & $\begin{array}{l}\text { After } 1 \text { month } \\
\text { of stimulation, } \\
\text { the participant } \\
\text { transitioned from } \\
\text { VS to MCS and EEG } \\
\text { showed increased } \\
\text { theta power; } \\
\text { after } 3 \text { months of } \\
\text { stimulation, PET } \\
\text { showed increased } \\
\text { metabolic activity in } \\
\text { the thalamus, basal } \\
\text { ganglia and multiple } \\
\text { regions of the } \\
\text { cerebral cortex }\end{array}$ & $\begin{array}{l}\text { Proof of } \\
\text { principle that } \\
\text { vagal nerve } \\
\text { stimulation } \\
\text { can lead to } \\
\text { modulation } \\
\text { of cortical } \\
\text { activity } \\
\text { and clinical } \\
\text { improvement } \\
\text { in chronic } \\
\text { VS/UWS }\end{array}$ \\
\hline $\begin{array}{l}\text { Sensory } \\
\text { stimulation }\end{array}$ & $\begin{array}{l}\text { Pape et al. } \\
(2015)^{269}\end{array}$ & $15 \mathrm{TBI}$ & $1-6$ months & $\begin{array}{l}5 \mathrm{VS} / \\
\text { UWS, } \\
10 \mathrm{MCS}\end{array}$ & $\begin{array}{l}\text { Double- } \\
\text { blind, parallel }\end{array}$ & $\begin{array}{l}\text { Comparison } \\
\text { of FAST and } \\
\text { placebo (silence) } \\
\text { applied for } \\
10 \text { min four } \\
\text { times per day } \\
\text { for } 6 \text { weeks }\end{array}$ & $\begin{array}{l}\text { Behavioural } \\
\text { improvements in both } \\
\text { FAST and placebo } \\
\text { groups; compared } \\
\text { with participants } \\
\text { receiving placebo, } \\
\text { participants receiving } \\
\text { FAST showed a } \\
\text { greater improvement } \\
\text { on the Coma/Near } \\
\text { Coma Scale; and } \\
\text { more functional MRI } \\
\text { activation in language } \\
\text { regions and whole } \\
\text { brain in response to } \\
\text { verbal stimuli }\end{array}$ & $\begin{array}{l}\text { Small sample } \\
\text { size }\end{array}$ \\
\hline
\end{tabular}

CRS-R, Coma Recovery Scale - Revised; DLPFC, dorsolateral prefrontal cortex; DoC, disorders of consciousness; FAST, familiar auditory sensory training; FDG, fluorodeoxyglucose; MCS, minimally conscious state; TBI, traumatic brain injury; VS/UWS, vegetative state/unresponsive wakefulness syndrome. 
are lacking at each stage of recovery ${ }^{285}$. We urge clinicians to embrace humility and acknowledge uncertainty when prognosticating. Behavioural, imaging and electrophysiological data should be integrated into a multimodal approach to prognostication, as no single tool accounts for the wide variance in outcomes for patients with DoC. New pharmacological and electrophysiological therapies provide hope that the future will bring not only more accurate prognostication but also new tools to promote recovery of consciousness ${ }^{273}$. It is unlikely that any single therapy will be effective for all patients; instead, we support a mechanistic approach to therapy selection that includes an individualized assessment of each patient's potential for a therapeutic response.

As investigational techniques and therapies begin to show clinical utility and efficacy, and as advanced imaging and electrophysiological techniques are incorporated into DoC guidelines ${ }^{2,182,205}$, clinicians are confronting new ethical questions about the rights of patients and their families. For example, whether or not all patients with DoC have the right to a comprehensive assessment with resting-state, stimulus-based and task-based fMRI and EEG, as well as PET, TMS-EEG and other diagnostic modalities, will need to be decided. If this kind of comprehensive assessment is performed in all patients, clinicians will need to decide whether these data should be acquired locally and sent to speciality centres for analysis, or whether patients should be transferred to speciality centres for data acquisition and analysis. These questions pertaining to patient rights and resource allocation are, at the present time, inextricably linked. Only a limited number of specialized centres currently have the infrastructure, resources and expertise to perform these assessments, but the expansion of open-source sharing of methods should make these assessments more generalizable in the future. Whether a diagnosis of CMD will lead to actionable therapies is currently unknown, but as data emerge suggesting that detection of CMD predicts functional recovery ${ }^{95}$, the ethical importance of including CMD assessments in discussions about goals of care becomes more compelling ${ }^{286}$.

Ethicists have now partnered with clinician and family stakeholders to develop consensus recommendations about the most ethically appropriate and clinically feasible approaches to implementation of advanced techniques for $\mathrm{CMD}$ detection ${ }^{287}$. These efforts towards CMD detection proceed with the recognition that many patients with severe brain injuries never recover consciousness and live with a persistent DoC. Nevertheless, as evidence is accumulating that some patients with DoC have the potential for recovery of consciousness and meaningful neurological function, and as the diagnostic label of 'permanent' DoC has now been abandoned in recognition of unexpected late recoveries ${ }^{2,182,205}$, clinicians should consider all possible diagnostic, prognostic and therapeutic approaches to support recovery of consciousness in patients with DoC.

Published online 14 December 2020
1. Giacino, J. T., Fins, J. J., Laureys, S. \& Schiff, N. D. Disorders of consciousness after acquired brain injury: the state of the science. Nat. Rev. Neurol. 10, 99-114 (2014).

2. Giacino, J. T. et al. Practice guideline update recommendations summary: disorders of consciousness: report of the Guideline Development, Dissemination, and Implementation Subcommittee of the American Academy of Neurology; the American Congress of Rehabilitation Medicine; and the National Institute on Disability, Independent Living, and Rehabilitation Research. Neurology 91, 450-460 (2018).

3. Teasdale, G. \& Jennett, B. Assessment of coma and impaired consciousness. A practical scale. Lancet $\mathbf{2}$ 81-84 (1974).

4. Laureys, S. et al. Unresponsive wakefulness syndrome: a new name for the vegetative state or apallic syndrome. BMC Med. 8, 68 (2010).

5. Jennett, B. \& Plum, F. Persistent vegetative state after brain damage. A syndrome in search of a name. Lancet 1, 734-737 (1972).

6. Multi-Society Task Force on PVS. Medical aspects of the persistent vegetative state (1). N. Engl. J. Med. 330, 1499-1508 (1994)

7. Giacino, J. T. et al. The minimally conscious state: definition and diagnostic criteria. Neurology 58 349-353 (2002)

8. Bruno, M. A., Vanhaudenhuyse, A., Thibaut, A. Moonen, G. $\&$ Laureys, S. From unresponsive wakefulness to minimally conscious PLUS and functional locked-in syndromes: recent advances in our understanding of disorders of consciousness. J. Neurol. 258, 1373-1384 (2011).

9. Thibaut, A., Bodien, Y. G., Laureys, S. \& Giacino, J. T. Minimally conscious state "plus": diagnostic criteria and relation to functional recovery. J. Neurol. 267, 1245-1254 (2020).

10. Giacino, J. T. et al. Behavioral recovery and early decision making in patients with prolonged disturbance in consciousness after traumatic brain injury. J. Neurotrauma 37, 357-365 (2020).

11. Schiff, N. D. Cognitive motor dissociation following severe brain injuries. JAMA Neurol. 72, 1413-1415 (2015).

12. Hemphill, J. C. 3rd \& White, D. B. Clinical nihilism in neuroemergencies. Emerg. Med. Clin. North Am. 27 27-37 (2009).
13. Leblanc, G. et al. Incidence and impact of withdrawal of life-sustaining therapies in clinical trials of severe traumatic brain injury: a systematic review. Clin. Trials 15, 398-412 (2018)

14. Elmer, J. et al. Association of early withdrawal of life-sustaining therapy for perceived neurological prognosis with mortality after cardiac arrest. Resuscitation 102, 127-135 (2016).

15. Posner, J. B., Saper, C. B., Schiff, N. D. \& Claassen, J. Plum and Posner's Diagnosis and Treatment of Stupor and Coma 5th edn (Oxford Univ. Press, 2019).

16. Parvizi, J. \& Damasio, A. R. Neuroanatomical correlates of brainstem coma. Brain 126, 1524-1536 (2003)

17. Fischer, D. B. et al. A human brain network derived from coma-causing brainstem lesions. Neurology 87 , 2427-2434 (2016).

18. Schiff, N. D. Resolving the role of the paramedian thalamus in forebrain arousal mechanisms. Ann. Neurol. 84, 812-813 (2018).

19. Steriade, M., Nunez, A. \& Amzica, F. A novel slow $(<1 \mathrm{~Hz})$ oscillation of neocortical neurons in vivo: depolarizing and hyperpolarizing components. J. Neurosci. 13, 3252-3265 (1993).

20. Timofeev, I., Grenier, F., Bazhenov, M., Sejnowski, T. J. $\&$ Steriade, M. Origin of slow cortical oscillations in deafferented cortical slabs. Cereb. Cortex 10 1185-1199 (2000)

21. Gold, L. \& Lauritzen, M. Neuronal deactivation explains decreased cerebellar blood flow in response to focal cerebral ischemia or suppressed neocortical function. Proc. Natl Acad. Sci. USA 99, 7699-7704 (2002).

22. Timofeev, I., Grenier, F. \& Steriade, M. Disfacilitation and active inhibition in the neocortex during the natural sleep-wake cycle: an intracellular study. Proc. Natl Acad. Sci. USA 98, 1924-1929 (2001).

23. Blumenfeld, $\mathrm{H}$. et al. Ictal neocortical slowing in temporal lobe epilepsy. Neurology 63, 1015-1021 (2004).

24. Brown, E. N., Lydic, R. \& Schiff, N. D. General anesthesia, sleep, and coma. N. Engl. J. Med. 363 2638-2650 (2010).

25. Fridman, E. A ., Beattie, B. J., Broft, A Laureys, S \& Schiff, N. D. Regional cerebral metabolic patterns demonstrate the role of anterior forebrain mesocircuit dysfunction in the severely injured brain. Proc. Natl Acad. Sci. USA 111, 6473-6478 (2014).
26. Stender, J. et al. The minimal energetic requirement of sustained awareness after brain injury. Curr. Biol. 26, 1494-1499 (2016).

27. Schiff, N. D. Recovery of consciousness after brain injury: a mesocircuit hypothesis. Trends Neurosci. 33, $1-9(2010)$

28. Laureys, S. \& Schiff, N. D. Coma and consciousness: paradigms (re)framed by neuroimaging. Neurolmage 61, 478-491 (2012)

29. Williams, S. T. et al. Common resting brain dynamics indicate a possible mechanism underlying zolpidem response in severe brain injury. eLife 2, e01157 (2013).

30. Vanhaudenhuyse, A. et al. Default network connectivity reflects the level of consciousness in non-communicative brain-damaged patients. Brain 133, 161-171 (2010).

31. Wu, X. et al. Intrinsic functional connectivity patterns predict consciousness level and recovery outcome in acquired brain injury. J. Neurosci. 35, 12932-12946 (2015).

32. Buckner, R. L. \& DiNicola, L. M. The brain's default network: updated anatomy, physiology and evolving insights. Nat. Rev. Neurosci. 20, 593-608 (2019).

33. Raichle, M. E. \& Snyder, A. Z. A default mode of brain function: a brief history of an evolving idea. Neurolmage 37, 1083-1090 (2007)

34. Seeley, W. W. et al. Dissociable intrinsic connectivity networks for salience processing and executive control. J. Neurosci. 27, 2349-2356 (2007).

35. Thibaut, A. et al. Clinical response to tDCS depends on residual brain metabolism and grey matter integrity in patients with minimally conscious state. Brain Stimul. 8, 1116-1123 (2015).

36. Threlkeld, Z. D. et al. Functional networks reemerge during recovery of consciousness after acute severe traumatic brain injury. Cortex 106, 299-308 (2018)

37. Lant, N. D., Gonzalez-Lara, L. E., Owen, A. M. \& Fernandez-Espejo, D. Relationship between the anterior forebrain mesocircuit and the default mode network in the structural bases of disorders of consciousness. Neuroimage Clin. 10, 27-35 (2016).

38. Redinbaugh, M. J. et al. Thalamus modulates consciousness via layer-specific control of cortex. Neuron 106, 66-75.e12 (2020). 
39. Schiff, N. D. Central lateral thalamic nucleus stimulation awakens cortex via modulation of cross-regional, laminar-specific activity during general anesthesia. Neuron 106, 1-3 (2020)

40. Schiff, N. D. Central thalamic contributions to arousal regulation and neurological disorders of consciousness. Ann. N. Y. Acad. Sci. 1129, 105-118 (2008).

41. Edlow, B. L. et al. Neuroanatomic connectivity of the human ascending arousal system critical to consciousness and its disorders. J. Neuropathol. Exp. Neurol. 71, 531-546 (2012).

42. Snider, S. B. et al. Disruption of the ascending arousal network in acute traumatic disorders of consciousness. Neurology 93, e1281-e1287 (2019)

43. Steriade, $M$. Arousal: revisiting the reticular activating system. Science 272, 225-226 (1996).

44. Moruzzi, G. \& Magoun, H. W. Brain stem reticular formation and activation of the EEG. Electroencephalogr. Clin. Neurophysiol. 1, 455-473 (1949).

45. Berlingeri, M., Magnani, F. G., Salvato, G., Rosanova, M. $\&$ Bottini, G. Neuroimaging studies on disorders of consciousness: a meta-analytic evaluation. J. Clin. Med. 8, 516 (2019).

46. Rudolph, M., Pelletier, J. G., Pare, D. \& Destexhe, A Characterization of synaptic conductances and integrative properties during electrically induced EEG-activated states in neocortical neurons in vivo. J. Neurophysiol. 94, 2805-2821 (2005).

47. Schiff, N. D. in Brain Function and Responsiveness in Disorders of Consciousness Ch. 15 (eds Monti, M. M. \& Sannita, W. G.) 195-204 (Springer International 2016).

48. Schiff, N. D., Nauvel, T. \& Victor, J. D. Large-scale brain dynamics in disorders of consciousness Curr. Opin. Neurobiol. 25, 7-14 (2014).

49. Becker, D. A. et al. A major miss in prognostication after cardiac arrest: burst suppression and brain healing. Epilepsy Behav. Case Rep. 7, 1-5 (2017)

50. Ching, S., Purdon, P. L., Vijayan, S., Kopell, N. J. \& Brown, E. N. A neurophysiological-metabolic model for burst suppression. Proc. Natl Acad. Sci. USA 109, 3095-3100 (2012).

51. Silva, L. R., Amitai, Y. \& Connors, B. W. Intrinsic oscillations of neocortex generated by layer 5 pyramidal neurons. Science 251, 432-435 (1991).

52. Llinas, R. R., Ribary, U., Jeanmonod, D., Kronberg, E. \& Mitra, P. P. Thalamocortical dysrhythmia: a neurological and neuropsychiatric syndrome characterized by magnetoencephalography. Proc. Nat Acad. Sci. USA 96, 15222-15227 (1999).

53. Llinas, R., Urbano, F. J., Leznik, E., Ramirez, R. R. \& van Marle, H. J. Rhythmic and dysrhythmic thalamocortical dynamics: GABA systems and the edge effect. Trends Neurosci. 28, 325-333 (2005).

54. Drover, J. D. \& Schiff, N. D. A method for decomposing multivariate time series into a causal hierarchy within specific frequency bands. J. Comput Neurosci. 45, 59-82 (2018)

55. Steriade, M., Timofeev, I. \& Grenier, F. Natural waking and sleep states: a view from inside neocortical neurons. J. Neurophysiol. 85, 1969-1985 (2001).

56. Forgacs, P. B. et al. Dynamic regimes of neocortical activity linked to corticothalamic integrity correlate with outcomes in acute anoxic brain injury after cardiac arrest. Ann. Clin. Transl Neurol. 4, 119-129 (2017).

57. Claassen, J. et al. Bedside quantitative electroencephalography improves assessment of consciousness in comatose subarachnoid hemorrhage patients. Ann. Neurol. 80, 541-553 (2016).

58. Shah, S. A. et al. Executive attention deficits after traumatic brain injury reflect impaired recruitment of resources. Neuroimage Clin. 14, 233-241 (2017).

59. Shah, S. A. et al. Focal electroencephalographic changes index post-traumatic confusion and outcome J. Neurotrauma 34, 2691-2699 (2017).

60. Chatelle, $\mathrm{C}$. et al. Changes in cerebral metabolism in patients with a minimally conscious state responding to zolpidem. Front. Hum. Neurosci. 8, 917 (2014).

61. Destexhe, A., Rudolph, M. \& Pare, D. The high-conductance state of neocortical neurons in vivo. Nat. Rev. Neurosci. 4, 739-751 (2003)

62. Dikmen, S. S. et al. Cognitive outcome following traumatic brain injury. J. Head. Trauma. Rehabil. 24, 430-438 (2009)

63. Newcombe, V. F. et al. Aetiological differences in neuroanatomy of the vegetative state: insights from diffusion tensor imaging and functional implications. J. Neurol. Neurosurg. Psychiatry 81, 552-561 (2010).

64. Hammond, F. M. et al. Disorders of consciousness due to traumatic brain injury: functional status ten years post-injury. J. Neurotrauma 36, 1136-1146 (2019).
65. Edlow, B. L. et al. Unexpected recovery of function after severe traumatic brain injury: the limits of early neuroimaging-based outcome prediction. Neurocrit Care 19, 364-375 (2013).

66. Edlow, B. L., Threlkeld, Z. D., Fehnel, K. P. \& Bodien, Y. G. Recovery of functional independence after traumatic transtentorial herniation with Duret hemorrhages. Front. Neurol. 10, 1077 (2019).

67. Muccio, C. F. et al. Reversible post-traumatic bilateral extensive restricted diffusion of the brain. A case study and review of the literature. Brain Inj. 23 466-472 (2009).

68. Stiver, S. I., Gean, A. D. \& Manley, G. T. Survival with good outcome after cerebral herniation and Duret hemorrhage caused by traumatic brain injury. J. Neurosurg. 110, 1242-1246 (2009).

69. Wijdicks, E. F. et al. Recommendations for the management of cerebral and cerebellar infarction with swelling: a statement for healthcare professionals from the American Heart Association/American Stroke Association. Stroke 45, 1222-1238 (2014).

70. Lord, A. S., Gilmore, E., Choi, H. A., Mayer, S. A. \& VISTA-ICH Collaboration. Time course and predictors of neurological deterioration after intracerebral hemorrhage. Stroke 46, 647-652 (2015)

71. Rosengart, A. J., Schultheiss, K. E., Tolentino, J. \& Macdonald, R. L. Prognostic factors for outcome in patients with aneurysmal subarachnoid hemorrhage. Stroke 38, 2315-2321 (2007)

72. Wijdicks, E. F. et al. Practice parameter: prediction of outcome in comatose survivors after cardiopulmonary resuscitation (an evidence-based review): report of the quality standards subcommittee of the American Academy of Neurology. Neurology 67, 203-210 (2006).

73. Hemphill, J. C. 3rd, Bonovich, D. C., Besmertis, L. Manley, G. T. \& Johnston, S. C. The ICH score: simple, reliable grading scale for intracerebral hemorrhage. Stroke 32, 891-897 (2001).

74. Turgeon, A. F. et al. Mortality associated with withdrawal of life-sustaining therapy for patients with severe traumatic brain injury: a Canadian multicentre cohort study. CMAJ 183, 1581-1588 (2011).

75. Peberdy, M. A. et al. Cardiopulmonary resuscitation of adults in the hospital: a report of 14720 cardiac arrests from the National Registry of Cardiopulmonary Resuscitation. Resuscitation 58, 297-308 (2003).

76. Izzy, S., Compton, R., Carandang, R., Hall, W. \& Muehlschlegel, S. Self-fulfilling prophecies through withdrawal of care: do they exist in traumatic brain injury, too? Neurocrit Care 19, 347-363 (2013).

77. Wijdicks, E. F., Bamlet, W. R., Maramattom, B. V. Manno, E. M. \& McClelland, R. L. Validation of a new coma scale: the FOUR score. Ann. Neurol. 58 , 585-593 (2005)

78. Foo, C. C., Loan, J. J. M. \& Brennan, P. M. The relationship of the FOUR score to patient outcome: a systematic review. J. Neurotrauma 36, 2469-2483 (2019).

79. Teasdale, G. M. et al. A universal subarachnoid hemorrhage scale: report of a committee of th World Federation of Neurosurgical Societies. J. Neurol. Neurosurg. Psychiatry 51, 1457 (1988).

80. de Oliveira Manoel, A. L. et al. Functional outcome after poor-grade subarachnoid hemorrhage: a single-center study and systematic literature review. Neurocrit Care 25, 338-350 (2016)

81. Rittenberger, J. C. Tisherman, S. A. Holm, M. B. Guyette, F. X. \& Callaway, C. W. An early, novel illness severity score to predict outcome after cardiac arrest. Resuscitation 82, 1399-1404 (2011).

82. Coppler, P. J. et al. Validation of the Pittsburgh Cardiac Arrest Category illness severity score. Resuscitation 89, 86-92 (2015).

83. Steyerberg, E. W. et al. Predicting outcome after traumatic brain injury: development and international validation of prognostic scores based on admission characteristics. PLoS Med. 5, e165 (2008).

84. Suys, T. et al. Automated quantitative pupillometry for the prognostication of coma after cardiac arrest. Neurocrit Care 21, 300-308 (2014).

85. Solari, D. et al. Early prediction of coma recovery after cardiac arrest with blinded pupillometry. Ann. Neurol. 81, 804-810 (2017)

86. Oddo, M. et al. Quantitative versus standard pupillary light reflex for early prognostication in comatose cardiac arrest patients: an international prospective multicenter double-blinded study. Intensive Care Med. 44, 2102-2111 (2018).

87. Maciel, C. B. et al. Corneal reflex testing in the evaluation of a comatose patient: an ode to precise semiology and examination skills. Neurocrit Care 33, 399-404 (2020).
88. Acosta, M. C., Tan, M. E., Belmonte, C. \& Gallar, J. Sensations evoked by selective mechanical, chemical, and thermal stimulation of the conjunctiva and cornea. Invest. Ophthalmol. Vis. Sci. 42, 2063-2067 (2001).

89. Greer, D. M. et al. Clinical examination for outcome prediction in nontraumatic coma. Crit. Care Med. 40 , 1150-1156 (2012).

90. Nolan, J. P. et al. European Resuscitation Council and European Society of Intensive Care Medicine 2015 guidelines for post-resuscitation care. Intensive Care Med. 41, 2039-2056 (2015).

91. Greer, D. M., Rosenthal, E. S. \& Wu, O. Neuroprognostication of hypoxic-ischaemic coma in the therapeutic hypothermia era. Nat. Rev. Neurol. 10, 190-203 (2014)

92. Giacino, J. T., Kalmar, K. \& Whyte, J. The JFK Coma Recovery Scale - Revised: measurement characteristics and diagnostic utility. Arch. Phys. Med. Rehabil. 85, 2020-2029 (2004).

93. Schnakers, C. et al. Diagnostic accuracy of the vegetative and minimally conscious state: clinical consensus versus standardized neurobehavioral assessment. BMC Neurol. 9, 35 (2009).

94. Giacino, J. T. \& Kalmar, K. The vegetative and minimally conscious states: a comparison of clinical features and functional outcome. J. Head. Trauma. Rehabil. 12, 36-51 (1997).

95. Claassen, J. et al. Detection of brain activation in unresponsive patients with acute brain injury. N. Engl. J. Med. 380, 2497-2505 (2019).

96. Faugeras, F. et al. Survival and consciousness recovery are better in the minimally conscious state than in the vegetative state. Brain Inj. 32, $72-77$ (2018).

97. Fins, J. J. Rights Come to Mind: Brain Injury, Ethics, and the Struggle for Consciousness (Cambridge Univ. Press, 2015)

98. Metter, R. B., Rittenberger, J. C., Guyette, F. X. \& Callaway, C. W. Association between a quantitative CT scan measure of brain edema and outcome after cardiac arrest. Resuscitation 82, 1180-1185 (2011).

99. Claassen, J. et al. Global cerebral edema after subarachnoid hemorrhage: frequency, predictors, and impact on outcome. Stroke 33, 1225-1232 (2002).

100. Gentry, L. R., Godersky, J. C., Thompson, B. \& Dunn, V. D. Prospective comparative study of intermediate-field MR and CT in the evaluation of closed head trauma. Am. J. Roentgenol. 150. 673-682 (1988).

101. Skandsen, T. et al. Prevalence and impact of diffuse axonal injury in patients with moderate and severe head injury: a cohort study of early magnetic resonance imaging findings and 1 -year outcome. J. Neurosurg. 113, 556-563 (2010).

102. Wu, O. et al. Comatose patients with cardiac arrest: predicting clinical outcome with diffusion-weighted MR imaging. Radiology 252, 173-181 (2009).

103. Wijman, C. A. et al. Prognostic value of brain diffusion-weighted imaging after cardiac arrest. Ann. Neurol. 65, 394-402 (2009).

104. Greer, D. M. et al. Hippocampal magnetic resonance imaging abnormalities in cardiac arrest are associated with poor outcome. J. Stroke Cerebrovasc. Dis. 22, 899-905 (2013).

105. Tong, K. A. et al. Diffuse axonal injury in children: clinical correlation with hemorrhagic lesions. Ann. Neurol. 56, 36-50 (2004).

106. Yanagawa, Y. et al. A quantitative analysis of head injury using $\mathrm{T} 2 *$-weighted gradient-echo imaging. J. Trauma. 49, 272-277 (2000).

107. Griffin, A. D. et al. Traumatic microbleeds suggest vascular injury and predict disability in traumatic brain injury. Brain 142, 3550-3564 (2019).

108. Izzy, S. et al. Revisiting grade 3 diffuse axonal injury: not all brainstem microbleeds are prognostically equal. Neurocrit Care 27, 199-207 (2017).

109. Edlow, B. L. et al. Disconnection of the ascending arousal system in traumatic coma. J. Neuropathol. Exp. Neurol. 72, 505-523 (2013).

110. McNab, J. A. et al. The human connectome project and beyond: initial applications of $300 \mathrm{mT} / \mathrm{m}$ gradients. Neurolmage 80, 234-245 (2013).

111. Smith, D. H., Hicks, R. \& Povlishock, J. T. Therapy development for diffuse axonal injury. J. Neurotrauma 30, 307-323 (2013)

112. Diaz-Arrastia, R. et al. Pharmacotherapy of traumatic brain injury: state of the science and the road forward: report of the Department of Defense Neurotrauma Pharmacology Workgroup. J. Neurotrauma 31 , 135-158 (2014).

113. Sair, H. I. et al. Early functional connectome integrity and 1-year recovery in comatose survivors of cardiac arrest. Radiology 287, 247-255 (2018). 
114. Koenig, M. A. et al. MRI default mode network connectivity is associated with functional outcome after cardiopulmonary arrest. Neurocrit Care $\mathbf{2 0}$ 348-357 (2014)

115. Norton, L. et al. Disruptions of functional connectivity in the default mode network of comatose patients. Neurology 78, 175-181 (2012).

116. Pugin, D. et al. Resting-state brain activity for early prediction outcome in postanoxic patients in a coma with indeterminate clinical prognosis. AJNR Am. $J$. Neuroradiol. 41, 1022-1030 (2020).

117. Silva, S. et al. Disruption of posteromedial large-scale neural communication predicts recovery from coma. Neurology 85, 2036-2044 (2015).

118. Velly, L. et al. Use of brain diffusion tensor imaging for the prediction of long-term neurological outcomes in patients after cardiac arrest: a multicentre, international, prospective, observational, cohor study. Lancet Neurol. 17, 317-326 (2018).

119. Galanaud, D. et al. Assessment of white matter injury and outcome in severe brain trauma: a prospective multicenter cohort. Anesthesiology 117, 1300-1310 (2012)

120. Basser, P. J. \& Pierpaoli, C. Microstructural and physiological features of tissues elucidated by quantitative-diffusion-tensor MRI. J. Magn. Reson. B 111, 209-219 (1996)

121. Wang, J. Y. et al. Longitudinal changes of structural connectivity in traumatic axonal injury. Neurology 77 818-826 (2011).

122. Edlow, B. L. et al. Diffusion tensor imaging in acuteto-subacute traumatic brain injury: a longitudinal analysis. BMC Neurol. 16, 2 (2016).

123. Warner, M. A. et al. Assessing spatial relationships between axonal integrity, regional brain volumes, and neuropsychological outcomes after traumatic axonal injury. J. Neurotrauma 27, 2121-2130 (2010).

124. Edlow, B. L. et al. Personalized connectome mapping to guide targeted therapy and promote recovery of consciousness in the intensive care unit. Neurocrit Care 33, 364-375 (2020)

125. Yue, J. K. et al. Transforming research and clinical knowledge in traumatic brain injury pilot: multicenter implementation of the common data elements for traumatic brain injury. J. Neurotrauma 30 1831-1844 (2013).

126. Maas, A. I. R. et al. Traumatic brain injury: integrated approaches to improve prevention, clinical care, and research. Lancet Neurol. 16, 987-1048 (2017).

127. Haacke, E. M. et al. Common data elements in radiologic imaging of traumatic brain injury. J. Magn. Reson. Imaging 32, 516-543 (2010).

128. Nichols, T. E. et al. Best practices in data analysis and sharing in neuroimaging using MRI. Nat. Neurosci. 20, 299-303 (2017)

129. Fox, M. D. Mapping symptoms to brain networks with the human connectome. N. Engl. J. Med. 379, 2237-2245 (2018)

130. Crossley, N. A. et al. The hubs of the human connectome are generally implicated in the anatomy of brain disorders. Brain 137, 2382-2395 (2014).

131. Achard S. et al. Hubs of brain functional networks are radically reorganized in comatose patients. Proc. Natl Acad. Sci. USA 109, 20608-20613 (2012).

132. Sharp, D. J., Scott, G. \& Leech, R. Network dysfunction after traumatic brain injury. Nat. Rev. Neurol. 10, 156-166 (2014).

133. Snider, S. B. et al. Cortical lesions causing loss of consciousness are anticorrelated with the dorsal brainstem. Hum. Brain Mapp. 41, 1520-1531 (2020)

134. Thengone, D. J., Voss, H. U., Fridman, E. A. \& Schiff, N. D. Local changes in network structure contribute to late communication recovery after severe brain injury. Sci. Transl Med. 8, 368re365 (2016)

135. Voss, H. U. et al. Possible axonal regrowth in late recovery from the minimally conscious state. J. Clin. Invest. 116, 2005-2011 (2006).

136. Bodien, Y. G., Chatelle, C. \& Edlow, B. L. Functional networks in disorders of consciousness. Semin. Neurol. 37, 485-502 (2017).

137. Kondziella, D. et al. Functional MRI for assessment of the default mode network in acute brain injury. Neurocrit Care 27, 401-406 (2017).

138. Fischer, D. et al. Intact brain network function in an unresponsive patient with COVID-19. Ann. Neurol. 88, 851-854 (2020)

139. Comanducci, A. et al. Basic and advanced neurophysiology in the prognostic and diagnostic evaluation of disorders of consciousness: review of an IFCN-endorsed expert group. Clin Neurophysiol. 131, 2736-2765 (2020).
140. Towne, A. R. et al. Prevalence of nonconvulsive status epilepticus in comatose patients. Neurology 54 340-345 (2000)

141. Claassen, J., Mayer, S. A., Kowalski, R. G., Emerson, R. G. \& Hirsch, L. J. Detection of electrographic seizures with continuous EEG monitoring in critically ill patients. Neurology 62 1743-1748 (2004).

142. Young, G. B., McLachlan, R. S., Kreeft, J. H. \& Demelo, J. D. An electroencephalographic classification for coma. Can. J. Neurol. Sci. 24 320-325 (1997)

143. Husari, K. S., Johnson, E. L. \& Ritzl, E. K. Acute and long-term outcomes of lateralized rhythmic delta activity (LRDA) versus lateralized periodic discharges (LPDs) in critically ill patients. Neurocrit. Care https:// doi.org/10.1007/s12028-020-01017-y (2020).

144. Tabaeizadeh, M. et al. Burden of epileptiform activity predicts discharge neurologic outcomes in severe acute ischemic stroke. Neurocrit Care 32, 697-706 (2020).

145. Oddo, M., Carrera, E., Claassen, J., Mayer, S. A. \& Hirsch, L. J. Continuous electroencephalography in the medical intensive care unit. Crit. Care Med. 37 2051-2056 (2009).

146. De Marchis, G. M. et al. Seizure burden in subarachnoid hemorrhage associated with functional and cognitive outcome. Neurology 86, 253-260 (2016).

147. Claassen, J. et al. Electrographic seizures and periodic discharges after intracerebral hemorrhage. Neurology 69, 1356-1365 (2007)

148. Zafar, S. F. et al. Effect of epileptiform abnormality burden on neurologic outcome and antiepileptic drug management after subarachnoid hemorrhage. Clin. Neurophysiol. 129, 2219-2227 (2018).

149. Rossetti, A. O., Rabinstein A. A. \& Oddo, M. Neurological prognostication of outcome in patients in coma after cardiac arrest. Lancet Neurol. 15 597-609 (2016)

150. Rossetti, A. O. et al. Electroencephalography predicts poor and good outcomes after cardiac arrest: a twocenter study. Crit. Care Med. 45, e674-e682 (2017).

151. Rossetti, A. O. Oddo, M. Liaudet, L. \& Kaplan, P. W. Predictors of awakening from postanoxic status epilepticus after therapeutic hypothermia. Neurology 72, 744-749 (2009).

152. Elmer, J. et al. Clinically distinct electroencephalographic phenotypes of early myoclonus after cardiac arrest. Ann. Neurol. 80, 175-184 (2016).

153. Bekinschtein, T. A. et al. Neural signature of the conscious processing of auditory regularities. Proc. Natl Acad. Sci. USA 106, 1672-1677 (2009).

154. Amorim, E. et al. Estimating the false positive rate of absent somatosensory evoked potentials in cardiac arrest prognostication. Crit. Care Med. 46, e1213-e1221 (2018).

155. Carter, B. G. \& Butt, W. Review of the use of somatosensory evoked potentials in the prediction of outcome after severe brain injury. Crit. Care Med. 29 178-186 (2001)

156. Forgacs, P. B. et al. Preservation of electroencephalographic organization in patients with impaired consciousness and imaging-based evidence of command-following. Ann. Neurol. 76, 869-879 (2014).

157. Estraneo, A. et al. Standard EEG in diagnostic process of prolonged disorders of consciousness. Clin. Neurophysiol. 127, 2379-2385 (2016).

158. Jorgensen, E. O. $\&$ Holm, S. The natural course of neurological recovery following cardiopulmonary resuscitation. Resuscitation 36, 111-122 (1998).

159. Engemann, D. A. et al. Robust EEG-based cross-site and cross-protocol classification of states of consciousness. Brain 141, 3179-3192 (2018).

160. Sitt, J. D. et al. Large scale screening of neural signatures of consciousness in patients in a vegetative or minimally conscious state. Brain 137, 2258-2270 (2014).

161. Gosseries, O et al. Automated EEG entropy measurements in coma, vegetative state/unresponsive wakefulness syndrome and minimally conscious state. Funct. Neurol. 26, 25-30 (2011)

162. Mikell, C. B. et al. Frontal networks associated with command following after hemorrhagic stroke. Stroke 46, 49-57 (2015)

163. Streitberger, K. J. et al. Neuron-specific enolase predicts poor outcome after cardiac arrest and targeted temperature management: a multicenter study on 1,053 patients. Crit. Care Med. 45 1145-1151 (2017)

164. Mattsson, N. et al. Serum Tau and neurological outcome in cardiac arrest. Ann. Neurol. 82, 665-675 (2017).
165. Moseby-Knappe, M. et al. Serum neurofilament light chain for prognosis of outcome after cardiac arrest. JAMA Neurol. 76, 64-71 (2019).

166. Owen, A. M. et al. Detecting awareness in the vegetative state. Science 313, 1402 (2006).

167. Schnakers, C. et al. Preserved covert cognition in noncommunicative patients with severe brain injury? Neurorehabil. Neural Repair. 29, 308-317 (2015).

168. Gosseries, O., Zasler, N. D. \& Laureys, S. Recent advances in disorders of consciousness: focus on the diagnosis. Brain Inj. 28, 1141-1150 (2014).

169. Edlow, B. L. et al. Early detection of consciousness in patients with acute severe traumatic brain injury. Brain 140, 2399-2414 (2017).

170. Bodien, Y. G., Giacino, J. T. \& Edlow, B. L. Functional MRI motor imagery tasks to detect command following in traumatic disorders of consciousness. Front. Neurol. 8, 688 (2017).

171. Kondziella, D., Friberg, C. K., Frokjaer, V. G. Fabricius, M. \& Moller, K. Preserved consciousness in vegetative and minimal conscious states: systematic review and meta-analysis. J. Neurol. Neurosurg. Psychiatry 87, 485-492 (2016).

172. Cruse, D. et al. Bedside detection of awareness in the vegetative state: a cohort study. Lancet $\mathbf{3 7 8}$, 2088-2094 (2011).

173. Bodien, Y. G., Threlkeld, Z. D. \& Edlow, B. L. Default mode network dynamics in covert consciousness. Cortex 117, 571-574 (2019)

174. Goldfine, A. M. et al. Reanalysis of "Bedside detection of awareness in the vegetative state: a cohort study". Lancet 381, 289-291 (2013).

175. Chatelle, C., Spencer, C. A., Cash, S. S., Hochberg, L. R. \& Edlow, B. L. Feasibility of an EEG-based braincomputer interface in the intensive care unit. Clin. Neurophysiol 129, 1519-1525 (2018).

176. Rohaut, B., Eliseyev, A. \& Claassen, J. Uncovering consciousness in unresponsive ICU patients: technical, medical and ethical considerations. Crit. Care 23, 78 (2019)

177. Menon, D. K. et al. Cortical processing in persistent vegetative state. Lancet 352, 200 (1998).

178. Schiff, N. D. \& Plum, F. Cortical function in the persistent vegetative state. Trends Cogn. Sci. 3 43-44 (1999)

179. Coleman, M. R. et al. Towards the routine use of brain imaging to aid the clinical diagnosis of disorders of consciousness. Brain 132, 2541-2552 (2009).

180. Fernandez-Espejo, D. et al. Cerebral response to speech in vegetative and minimally conscious states after traumatic brain injury. Brain Inj. 22, 882-890 (2008)

181. Di, H. B. et al. Cerebral response to patient's own name in the vegetative and minimally conscious states. Neurology 68, 895-899 (2007).

182. Kondziella, D. et al. European Academy of Neurology guideline on the diagnosis of coma and other disorders of consciousness. Eur. J. Neurol. 27 741-756 (2020).

183. Braiman, C. et al. Cortical response to the natura speech envelope correlates with neuroimaging evidence of cognition in severe brain injury. Curr. Biol. 28, 3833-3839.e3 (2018).

184. Chatelle, C. et al. EEG correlates of language function in traumatic disorders of consciousness. Neurocrit Care 33, 449-457 (2020).

185. Macdonald, R. L. Delayed neurological deterioration after subarachnoid haemorrhage. Nat. Rev. Neurol. 10, 44-58 (2014)

186. Diringer, M. N. et al. Critical care management of patients following aneurysmal subarachnoid hemorrhage: recommendations from the Neurocritical Care Society's Multidisciplinary Consensus Conference. Neurocrit Care 15, 211-240 (2011).

187. Bernard, S. A. et al. Treatment of comatose survivors of out-of-hospital cardiac arrest with induced hypothermia. N. Engl. J. Med. 346, 557-563 (2002).

188. Nielsen, N. et al. Targeted temperature management at $33^{\circ} \mathrm{C}$ versus $36^{\circ} \mathrm{C}$ after cardiac arrest. N. Engl. J. Med. 369, 2197-2206 (2013).

189. Lascarrou, J. B. et al. Targeted temperature management for cardiac arrest with nonshockable rhythm. N. Engl. J. Med. 381, 2327-2337 (2019)

190. Andrews, P. J. et al. Hypothermia for intracranial hypertension after traumatic brain injury. N. Engl. J. Med. 373, 2403-2412 (2015).

191. Cooper, D. J. et al. Effect of early sustained prophylactic hypothermia on neurologic outcomes among patients with severe traumatic brain injury: the POLAR randomized clinical trial. JAMA 320 2211-2220 (2018). 
192. Clifton, G. L. et al. Lack of effect of induction of hypothermia after acute brain injury. N. Engl. J. Med. 344, 556-563 (2001).

193. Dietrich, W. D. \& Bramlett, H. M. Therapeutic hypothermia and targeted temperature management in traumatic brain injury: clinical challenges for successful translation. Brain Res. 1640, 94-103 (2016).

194. Hutchinson, P. J. et al. Trial of decompressive craniectomy for traumatic intracranial hypertension N. Engl. J. Med. 375, 1119-1130 (2016).

195. Meythaler, J. M., Brunner, R. C., Johnson, A. \& Novack, T. A. Amantadine to improve neurorecovery in traumatic brain injury-associated diffuse axonal injury: a pilot double-blind randomized trial. J. Head. Trauma. Rehabil. 17, 300-313 (2002).

196. Ghalaenovi, H. et al. The effects of amantadine on traumatic brain injury outcome: a double-blind, randomized, controlled, clinical trial. Brain Inj. 32, 1050-1055 (2018)

197. Barra, M. E. et al. Stimulant therapy in acute traumatic brain injury: prescribing patterns and adverse event rates at 2 Level 1 trauma centers. J. Intensive. Care Med. 35, 11196-1202 (2020).

198. Alkhachroum, A. et al. EEG to detect early recovery of consciousness in amantadine-treated acute brain injury patients. J. Neurol. Neurosurg. Psychiatry 91 675-676 (2020)

199. Monti, M. M., Schnakers, C., Korb, A. S., Bystritsky, A $\&$ Vespa, P. M. Non-invasive ultrasonic thalamic stimulation in disorders of consciousness after severe brain injury: a first-in-man report. Brain Stimul. 9 , 940-941 (2016)

200. American Congress of Rehabilitation Medicine, Brain Injury-Interdisciplinary Special Interest Group, Disorders of Consciousness Task Force, et al. Assessment scales for disorders of consciousness: evidence-based recommendations for clinical practice and research. Arch. Phys. Med. Rehabil. 91, 1795-1813 (2010)

201. Wannez, S. et al. The repetition of behavioral assessments in diagnosis of disorders of consciousness. Ann. Neurol. 81, 883-889 (2017).

202. Pincherle, A. et al. Motor behavior unmasks residua cognition in disorders of consciousness. Ann. Neurol. 85, 443-447 (2019).

203. Johr, J. et al. Recovery in cognitive motor dissociation after severe brain injury: a cohort study. PLOS ONE 15, e0228474 (2020).

204. Estraneo, A. et al. Late recovery after traumatic anoxic, or hemorrhagic long-lasting vegetative state. Neurology 75, 239-245 (2010).

205. Giacino, J. T. et al. Practice guideline update recommendations summary: disorders of consciousness: Report of the Guideline Development, Dissemination, and Implementation Subcommittee of the American Academy of Neurology; the American Congress of Rehabilitation Medicine; and the National Institute on Disability, Independent Living, and Rehabilitation Research. Arch. Phys. Med. Rehabil. 99, 1699-1709 (2018)

206. Laureys, S. et al. Impaired effective cortical connectivity in vegetative state: preliminary investigation using PET. Neurolmage 9, 377-382 (1999).

207. Laureys, S. et al. Restoration of thalamocortical connectivity after recovery from persistent vegetative state. Lancet 355, 1790-1791 (2000).

208. Owen, A. M. et al. Residual auditory function in persistent vegetative state: a combined PET and fMR study. Neuropsychol. Rehabil. 15, 290-306 (2005)

209. Sharp, D. J. et al. Default mode network functional and structural connectivity after traumatic brain injury. Brain 134, 2233-2247 (2011).

210. Hillary, F. G. et al. Changes in resting connectivity during recovery from severe traumatic brain injury. Int. J. Psychophysiol. 82, 115-123 (2011).

211. Bonnelle, V. et al. Default mode network connectivity predicts sustained attention deficits after traumatic brain injury. J. Neurosci. 31, 13442-13451 (2011).

212. Bonnelle, V. et al. Salience network integrity predicts default mode network function after traumatic brain injury. Proc. Natl Acad. Sci. USA 109, 4690-4695 (2012).

213. Cauda, F. et al. Disrupted intrinsic functional connectivity in the vegetative state. J. Neurol Neurosurg. Psychiatry 80, 429-431 (2009).

214. Soddu, A. et al. Identifying the default-mode component in spatial IC analyses of patients with disorders of consciousness. Hum. Brain Mapp. 33 778-796 (2012)

215. Demertzi, A. et al. Intrinsic functional connectivity differentiates minimally conscious from unresponsive patients. Brain 138, 2619-2631 (2015).
216. Demertzi, A. et al. Multiple fMRI system-level baseline connectivity is disrupted in patients with consciousness alterations. Cortex 52, 35-46 (2014).

217. Qin, P. et al. How are different neural networks related to consciousness? Ann. Neurol. 78, 594-605 (2015).

218. Song, M. et al. Prognostication of chronic disorders of consciousness using brain functional networks and clinical characteristics. eLife 7, e36173 (2018).

219. Fernandez-Espejo, D. et al. A role for the default mode network in the bases of disorders of consciousness. Ann. Neurol. 72, 335-343 (2012).

220. Golland, Y. et al. Extrinsic and intrinsic systems in the posterior cortex of the human brain revealed during natural sensory stimulation. Cereb. Cortex 17 766-777 (2007)

221. Fox, M. D. \& Raichle, M. E. Spontaneous fluctuations in brain activity observed with functional magnetic resonance imaging. Nat. Rev. Neurosci. 8, 700-711 (2007).

222. Fox, M. D. et al. The human brain is intrinsically organized into dynamic, anticorrelated functional networks. Proc. Natl Acad. Sci. USA 102, 9673-9678 (2005).

223. Demertzi, A. et al. Human consciousness is supported by dynamic complex patterns of brain signal coordination. Sci. Adv. 5, eaat7603 (2019).

224. Brooks, J C., Faull, O. K., Pattinson, K. T. \& Jenkinson, M. Physiological noise in brainstem FMRI. Front. Hum. Neurosci. 7, 623 (2013)

225. Beissner, F., Schumann, A., Brunn, F., Eisentrager, D. \& Bar, K. J. Advances in functional magnetic resonance imaging of the human brainstem. Neurolmage $\mathbf{8 6}$ 91-98 (2014)

226. Bianciardi, M. et al. In vivo functional connectome of human brainstem nuclei of the ascending arousal, autonomic, and motor systems by high spatial resolution 7-Tesla fMRI. MAGMA 29, 451-462 (2016)

227. Bar, K. J. et al. Functional connectivity and network analysis of midbrain and brainstem nuclei. Neurolmage 134, 53-63 (2016)

228. Curley, W. H., Forgacs, P. B., Voss, H. U., Conte, M. M $\Sigma$ Schiff, N. D. Characterization of EEG signals revealing covert cognition in the injured brain. Brain 141, 1404-1421 (2018)

229. Rosanova, M. et al. Sleep-like cortical OFF-periods disrupt causality and complexity in the brain of unresponsive wakefulness syndrome patients. Nat. Commun. 9, 4427 (2018).

230. Arnaldi, D. et al. The prognostic value of sleep patterns in disorders of consciousness in the sub-acute phase. Clin. Neurophysiol. 127, 1445-1451 (2016).

231. Kang, X. G. et al. Development of a simple score to predict outcome for unresponsive wakefulness syndrome. Crit. Care 18, R37 (2014).

232. Chennu, S. et al. Brain networks predict metabolism, diagnosis and prognosis at the bedside in disorders of consciousness. Brain 140, 2120-2132 (2017).

233. Schomer, D. L. \& Lopes da Silva, F. H. Niedermeyer's Electroencephalography: Basic Principles, Clinical Applications, and Related Fields 7th edn (Oxford Univ. Press, 2017).

234. Boly, M. et al. Preserved feedforward but impaired top-down processes in the vegetative state. Science 332, 858-862 (2011).

235. Kotchoubey, B. et al. Information processing in severe disorders of consciousness: vegetative state and minimally conscious state. Clin. Neurophysiol. 116 2441-2453 (2005)

236. Cavinato, M. et al. Post-acute P300 predicts recovery of consciousness from traumatic vegetative state. Brain Inj. 23, 973-980 (2009).

237. Daltrozzo, J., Wioland, N., Mutschler, V. \& Kotchoubey, B. Predicting coma and other low responsive patients outcome using event-related brain potentials: a meta-analysis. Clin. Neurophysiol. 118 606-614 (2007)

238. Steppacher, I. et al. N400 predicts recovery from disorders of consciousness. Ann. Neurol. 73 594-602 (2013)

239. Garrido, M. I., Kilner, J. M., Stephan, K. E. \& Friston, K. J. The mismatch negativity: a review of underlying mechanisms. Clin. Neurophysiol. 120 453-463 (2009).

240. Qin, P. et al. Mismatch negativity to the patient's own name in chronic disorders of consciousness. Neurosci. Lett. 448, 24-28 (2008).

241. Tzovara, A. et al. Prediction of awakening from hypothermic postanoxic coma based on auditory discrimination. Ann. Neurol. 79, 748-757 (2016).

242. Raimondo, F. et al. Brain-heart interactions reveal consciousness in noncommunicating patients. Ann. Neurol. 82, 578-591 (2017).
243. O'Kelly, J. et al. Neurophysiological and behavioral responses to music therapy in vegetative and minimally conscious states. Front. Hum. Neurosci. 7, 884 (2013).

244. Casali, A. G. et al. A theoretically based index of consciousness independent of sensory processing and behavior. Sci. Transl Med. 5, 198ra105 (2013).

245. Casarotto, S. et al. Stratification of unresponsive patients by an independently validated index of brain complexity. Ann. Neurol. 80, 718-729 (2016)

246. Tononi, G., Boly, M., Massimini, M. \& Koch, C. Integrated information theory: from consciousness to its physical substrate. Nat. Rev. Neurosci. 17 450-461 (2016).

247. Comolatti, R. et al. A fast and general method to empirically estimate the complexity of brain responses to transcranial and intracranial stimulations. Brain Stimul. 12, 1280-1289 (2019).

248. Belardinelli, P. et al. Reproducibility in TMS-EEC studies: a call for data sharing, standard procedures and effective experimental control. Brain Stimul. 12, 787-790 (2019).

249. Monti, M. M. et al. Willful modulation of brain activity in disorders of consciousness. N. Engl. J. Med. 362 , 579-589 (2010)

250. Stender, J. et al. Diagnostic precision of PET imaging and functional MRI in disorders of consciousness: a clinical validation study. Lancet 384, 514-522 (2014)

251. Goldfine, A. M., Victor, J. D., Conte, M. M., Bardin, J. C $\&$ Schiff, N. D. Determination of awareness in patients with severe brain injury using EEG power spectral analysis. Clin. Neurophysiol. 122, 2157-2168 (2011).

252. Monti, M. M., Pickard, J. D. \& Owen, A. M. Visual cognition in disorders of consciousness: from $\mathrm{V} 1$ to top-down attention. Hum. Brain Mapp. 34, 1245-1253 (2013)

253. Bardin, J. C. et al. Dissociations between behavioural and functional magnetic resonance imaging-based evaluations of cognitive function after brain injury. Brain 134, 769-782 (2011).

254. Naci, L. \& Owen, A. M. Making every word count for nonresponsive patients. JAMA Neurol. 70, 1235-1241 (2013).

255. Gibson, R. M. et al. Multiple tasks and neuroimaging modalities increase the likelihood of detecting covert awareness in patients with disorders of consciousness. Front. Hum. Neurosci. 8, 950 (2014).

256. Pisa, F. E., Biasutti, E., Drigo, D. \& Barbone, F. The prevalence of vegetative and minimally conscious states: a systematic review and methodological appraisal. J. Head. Trauma. Rehabil. 29, E23-E30 (2014).

257. van Erp, W. S. et al. The vegetative state/unresponsive wakefulness syndrome: a systematic review of prevalence studies. Eur. J. Neurol. 21, 1361-1368 (2014).

258. Di Perri, C. et al. Neural correlates of consciousness in patients who have emerged from a minimally conscious state: a cross-sectional multimodal imaging study. Lancet Neurol. 15, 830-842 (2016).

259. Iotzov, I. et al. Divergent neural responses to narrative speech in disorders of consciousness. Ann. Clin. Transl Neurol. 4, 784-792 (2017).

260. Fridman, E. A. \& Schiff, N. D. Neuromodulation of the conscious state following severe brain injuries. Curr. Opin. Neurobiol. 29, 172-177 (2014).

261. Giacino, J. T. et al. Placebo-controlled trial of amantadine for severe traumatic brain injury. N. Engl. Med. 366, 819-826 (2012).

262. Kim, Y. W. Shin, J. C. \& An, Y. S. Effects of methylphenidate on cerebral glucose metabolism in patients with impaired consciousness after acquired brain injury. Clin. Neuropharmacol. 32, 335-339 (2009)

263. Krimchansky, B. Z., Keren, O., Sazbon, L. ¿ Groswasser, Z. Differential time and related appearance of signs, indicating improvement in the state of consciousness in vegetative state traumatic brain injury (VS-TBI) patients after initiation of dopamine treatment. Brain Inj. 18, 1099-1105 (2004)

264. Passler, M. A. \& Riggs, R. V. Positive outcomes in traumatic brain injury-vegetative state: patients treated with bromocriptine. Arch. Phys. Med. Rehabil. 82, 311-315 (2001).

265. Fridman, E. A. et al. Continuous subcutaneous apomorphine for severe disorders of consciousness after traumatic brain injury. Brain Inj. 24, 636-641 (2010).

266. Manganotti, P. et al. Effect of high-frequency repetitive transcranial magnetic stimulation on brain excitability in severely brain-injured patients in minimally conscious or vegetative state. Brain Stimul. 6 , 913-921 (2013) 
267. Thibaut, A., Bruno, M. A., Ledoux, D., Demertzi, A. \& Laureys, S. tDCS in patients with disorders of consciousness: sham-controlled randomized double-blind study. Neurology 82, 1112-1118 (2014).

268. Corazzol, M. et al. Restoring consciousness with vagus nerve stimulation. Curr. Biol. 27, R994-R996 (2017).

269. Pape, T. L. et al. Placebo-controlled trial of familiar auditory sensory training for acute severe traumatic brain injury: a preliminary report. Neurorehabil Neural Repair. 29, 537-547 (2015).

270. Schnakers, C., Magee, W. L. \& Harris, B. Sensory stimulation and music therapy programs for treating disorders of consciousness. Front. Psychol. 7, 297 (2016).

271. Whyte, J. et al. Zolpidem and restoration of consciousness. Am. J. Phys. Med. Rehabil. 93, 101-113 (2014).

272. Schiff, N. D. et al. Behavioural improvements with thalamic stimulation after severe traumatic brain injury. Nature 448, 600-603 (2007).

273. Provencio, J. J. et al. The Curing Coma Campaign: framing initial scientific challenges - proceedings of the first Curing Coma Campaign Scientific Advisory Council Meeting. Neurocrit Care 33, 1-12 (2020).

274. Jenkins, P. O. et al. Stratifying drug treatment of cognitive impairments after traumatic brain injury using neuroimaging. Brain 142, 2367-2379 (2019).

275. Fridman, E. A., Osborne, J. R., Mozley, P. D., Victor, J. D \& Schiff, N. D. Presynaptic dopamine deficit in minimally conscious state patients following traumatic brain injury. Brain 142, 1887-1893 (2019).

276. Simon, D. W. et al. The far-reaching scope of neuroinflammation after traumatic brain injury. Nat. Rev. Neurol. 13, 171-191 (2017).

277. Shlosberg, D., Benifla, M., Kaufer, D. \& Friedman, A. Blood-brain barrier breakdown as a therapeutic target in traumatic brain injury. Nat. Rev. Neurol. 6, 393-403 (2010)

278. Johnson, V. E. et al. Inflammation and white matter degeneration persist for years after a single traumatic brain injury. Brain 136, 28-42 (2013).

279. Scott, G. et al. Minocycline reduces chronic microglial activation after brain trauma but increases neurodegeneration. Brain 141, 459-471 (2018).

280. Edlow, B. L. et al. Multimodal characterization of the late effects of traumatic brain injury: a methodological overview of the Late Effects of Traumatic Brain Injury project. J. Neurotrauma 35, 1604-1619 (2018).

281. Walker, W. C. et al. The Chronic Effects of Neurotrauma Consortium (CENC) multi-centre observational study: description of study and characteristics of early participants. Brain Inj. 30 1469-1480 (2016).

282. Mez, J. et al. Assessing clinicopathological correlation in chronic traumatic encephalopathy: rationale and methods for the UNITE study. Alzheimers Res. Ther. 7 , 62 (2015).

283. Smith, D. H., Johnson, V. E., Trojanowski, J. Q. \& Stewart, W. Chronic traumatic encephalopathy confusion and controversies. Nat. Rev. Neurol. 15 179-183 (2019).

284. Schiff, N. D. et al. in Fifth Annual Brain Initiative Investigators Meeting Abstract book [abstract S-124]. 250 (National Institute of Mental Health, 2019).

285. Kotchoubey, B. \& Pavlov, Y. G. A systematic review and meta-analysis of the relationship between brain data and the outcome in disorders of consciousness. Front. Neurol. 9, 315 (2018).

286. Edlow, B. L. \& Fins, J. J. Assessment of covert consciousness in the intensive care unit: clinical and ethical considerations. J. Head. Trauma. Rehabil. 33, 424-434 (2018)

287. Fins, J. J. \& Bernat, J. L. Ethical, palliative, and policy considerations in disorders of consciousness. Neurology 91, 471-475 (2018)

288. Cincotta, M. et al. No effects of $20 \mathrm{~Hz}-\mathrm{rTMS}$ of the primary motor cortex in vegetative state: a randomised, sham-controlled study. Cortex 71 368-376 (2015)

289. Parvizi, J. ¿ Damasio, A. Consciousness and the brainstem. Cognition 79, 135-160 (2001).

290. Baker, J. L. et al. Robust modulation of arousal regulation, performance, and frontostriatal activity through central thalamic deep brain stimulation in healthy nonhuman primates. J. Neurophysiol. 116 , 2383-2404 (2016).

291. Liu, J. et al. Frequency-selective control of cortical and subcortical networks by central thalamus. eLife $\mathbf{4}$, e09215 (2015).

292. Bernander, O., Douglas, R. J., Martin, K. A. \& Koch, C. Synaptic background activity influences spatiotemporal integration in single pyramidal cells. Proc. Natl Acad. Sci. USA 88, 11569-11573 (1991).
293. Thibaut, A., Schiff, N., Giacino, J., Laureys, S. \& Gosseries, O. Therapeutic interventions in patients with prolonged disorders of consciousness. Lancet Neurol. 18, 600-614 (2019).

294. Edlow, B. L. et al. 7 Tesla MRI of the ex vivo human brain at 100 micron resolution. Sci. Data 6, 244 (2019).

\section{Acknowledgements}

The authors' work is supported by the National Institutes of Health (NIH) National Institute of Neurological Disorders and Stroke (R01NS102574, R01NS106014, R03NS112760, R21NS109627, RF1NS115268, UH3NS95554), NIH Director's Office (DP2HD101400), James S. McDonnell Foundation, DANA Foundation, Jerold B. Katz and Lenny Katz Foundations, Rappaport Foundation and Tiny Blue Dot Foundation. The authors thank S.B. Snider for assistance with the creation of Fig. 3.

\section{Author contributions}

The authors contributed equally to all aspects of the manuscript.

\section{Competing interests}

The authors declare no competing interests.

Peer review information

Nature Reviews Neurology thanks R. Geocardin, J. Pickard, $\mathrm{M}$. Oddo and the other, anonymous, reviewer(s) for their contribution to the peer review of this work.

\section{Publisher's note}

Springer Nature remains neutral with regard to jurisdictional claims in published maps and institutional affiliations.

\section{Review criteria}

We selected articles for this Review from a MEDLINE search and from our personal reference libraries. The MEDLINE search included the following terms: 'disorders of consciousness', 'coma', 'vegetative state', 'unresponsive wakefulness syndrome', 'minimally conscious state', 'confusional state', 'cognitive motor dissociation', 'covert consciousness', 'neurologic examination', 'prognosis', 'functional MRI', 'diffusion tensor imaging', 'EEG', 'evoked potentials', 'P300' and 'mismatch negativity'. The search was from 2006 to 2020 and was exclusive to English language publications. Full-text papers were used for further leads.

(c) Springer Nature Limited 2020 